\title{
An Optimization Problem for Quadcopter Reference Flight Trajectory Generation
}

\author{
Karam Eliker $\mathbb{D}^{1},{ }^{1}$ Guoqing Zhang, ${ }^{1}$ Said Grouni, ${ }^{2}$ and Weidong Zhang $\mathbb{D}^{1,3}$ \\ ${ }^{1}$ Department of Automation, Shanghai Jiao Tong University, Shanghai 200240, China \\ ${ }^{2}$ Faculty of Sciences Engineering, University of Boumerdes, 35000 Boumerdes, Algeria \\ ${ }^{3}$ School of Computer Engineering and Science, Shanghai University, Shanghai 200444, China \\ Correspondence should be addressed to Weidong Zhang; wdzhang@sjtu.edu.cn
}

Received 22 October 2017; Accepted 29 March 2018; Published 26 July 2018

Academic Editor: Howard Li

Copyright (C) 2018 Karam Eliker et al. This is an open access article distributed under the Creative Commons Attribution License, which permits unrestricted use, distribution, and reproduction in any medium, provided the original work is properly cited.

\begin{abstract}
This paper deals with the reference flight trajectory generation and planning problems for quadcopter Unmanned Aerial Vehicle (UAV). The reference flight trajectory is defined as the composition of path and motion functions. Both of them are generated by using quintic B-spline functions. Based on differential flatness approach, the quadcopter dynamical constraints are satisfied instantaneously by computing the induced aerodynamical moments and lift force. The optimal reference flight trajectory, with respect to the mission requirements and imposed constraints, is reached by manipulating the control points' vectors of B-spline functions via a nonlinear constrained optimization method. The mission requirements are defined as a set of waypoints with their respective scheduled flight timetable. A minimum-energy cost function is developed to minimize the consumed energy and induced efforts by reference flight trajectory. For the need of the optimal overfly-times schedule, the overfly times with respect to the defined constraints and performance criteria are calculated. Numerical simulation results show the feasibility and effectiveness of the proposed optimization method.
\end{abstract}

\section{Introduction}

With a vast proliferation of Manned Aerial Vehicles (MAVs) and Unmanned Aerial Vehicles (UAVs), world air transportation traffic has witnessed a continuous increase causing a saturation in the airspace. The formal approach adopted by researchers to design the UAVs is to separate the avionics systems into three distinct and independent systems, which are Guidance, Navigation, and Control (GNC). By using this structure, UAVs could perform a variety of planning missions in either military or civilian fields. However, there are many incidents like a collision with civil aviation aircraft, invasion of privacy, and threats to security reported by [1] Federal Aviation Administration (FAA). These incidents are because of the lack of transportation rules and regulations supporting UAVs and the weakness current structure UAVs. Therefore, the need to integrate the UAVs in National Airspace System (NAS) is highly recommended.
To achieve this challenge, an adequate flight trajectory planner that could be embedded on quadcopter UAV should be developed. The flight trajectory planner could reduce uncertainty and increase predictability for Air Traffic Management (ATM). Planning missions in terms of waypoints with overfly times at which the UAV is scheduled to overfly could be a challenging problem for trajectory generation. Most of the previous works have focused on 3D trajectory planning problem and consider only waypoints without any overfly-times constraints. By contrast, this paper proposes a new optimization method to generate the reference flight trajectory that could fulfill some related requirements of NAS. The proposed flight trajectory planning should generate a trajectory that complies with the considered quadcopter dynamical constraints and meets the assigned missions. The assigned missions contain a set of waypoints with their respective scheduled flight timetable. For the necessity of optimal overfly-times schedule, the overfly times can be calculated in optimal way while considering the assigned 
constraints and performance criteria. By generating such reference flight trajectory, different tasks such as delivering and reconnaissance missions at acceptable overfly times could be performed. Moreover, the traffic managements for UAV could be enhanced by managing the scheduled flight timetable of waypoints.

Several papers of 3D trajectory planning problems while considering waypoints as physical points in the workspace have been done. On one hand, the authors in [2-7] proposed an optimization approach to solve Time Optimal Motion Planning (TOMP) between two configuration points by using a Nonlinear Programming (NLP) method. On the other hand, the authors in [8-10] have proposed an algorithm based on Pontryagin's minimum principle to solve TOMP between two states. In [11], the authors presented an approach that could link separate trajectories to form smooth analytically defined paths while satisfying a set of waypoint constraints. Moreover, in [12-14], a minimum snap cost function was designed to generate smooth trajectory while satisfying a sequence of consecutive waypoints. In [15], a quasi-optimal trajectory generation was developed to satisfy constraints concerning waypoints and their overfly times while minimizing the trajectory length.

Moreover, some papers have dealt with the minimumenergy problem. In [16], a quasi-optimal trajectory planner was proposed to generate trajectory between two points. The objective function was defined as a fuel consumption problem by considering the cost function as an average velocity. The authors in [17] supposed that the consumed energy was proportional to the rotors velocities. In [18], the authors designed a trajectory planning function for a smallscale helicopter in autorotation which could minimize the battery power consumption and avoid bang-bang type solutions. Nonlinear Programming problem was used to compute the optimal polynomial coefficients of the trajectory while minimizing the cost function written as the rate of all forces and moments applied on helicopter. In [19], a minimumenergy trajectory generation approach was proposed by defining the cost function as a function of the voltage and current across the four rotors. The similarity among these papers was the reference trajectory planning problems, defined as longitudinal, lateral, and altitude displacements, as NLP problem between two configuration points, while the difference was related to the parameterization of the reference trajectory.

The objective of this paper is the reference trajectory generation and planning problems for quadrotor UAV. The reference flight trajectory is defined as a composition of path and monotonically increasing motion functions. Both of them are generated by using quintic B-spline functions. Based on differential flatness approach, the quadcopter dynamical constraints are checked instantaneously by computing the induced aerodynamical roll and pitch, heading moments, and lift force, respectively. The optimal reference trajectory, which consists of longitudinal, lateral, and altitude displacements, in addition to yaw angle, is reached by manipulating the control points' vectors of B-spline functions via a nonlinear constrained optimization method while taking into account the mission requirements and defined constraints. The mission requirements are defined as a sequence of waypoints with their respective scheduled flight timetable, and each waypoint could represent a particular posture. A minimum-energy cost function is designed to enhance the performance criteria regarding trajectory length, induced efforts, consumed energy, and trajectory smoothness. For the optimal overflytimes schedule requirement, the overfly times are calculated by the proposed optimization method with regard to the interested constraints and performance criteria. Several configurations are studied and numerical simulation results show the feasibility and effectiveness of the proposed optimization method.

\section{Quadcopter Flight Dynamics}

The quadcopter UAV is a vertical take-off and landing (VTOL) aircraft. Its main characteristics are the ability of hovering, vertical take-off, and landing in any terrain. The displacement and rotation motions are achieved by adjusting the angular speed of each rotor. To design the reference flight trajectory, a simplified quadcopter flight dynamics are used to reduce the computation burden and to facilitate the optimization problem implementation. By considering that the quadcopter UAV performs angular motions of low amplitude and omitting the drag force, aerodynamical frictions, and gyroscopic effects moments, the simplified dynamic model of quadcopter UAV, in contrast to full quadcopter dynamics presented in $[21,22]$, is given by

$$
\begin{aligned}
& \ddot{\phi}=\frac{1}{I_{x}} U_{\phi} \\
& \ddot{\theta}=\frac{1}{I_{y}} U_{\theta} \\
& \ddot{\psi}=\frac{1}{I_{z}} U_{\psi} \\
& \ddot{x}=\frac{U_{z}}{m}(\cos \phi \sin \theta \cos \psi+\sin \phi \sin \psi) \\
& \ddot{y}=\frac{U_{z}}{m}(\cos \phi \sin \theta \sin \psi-\sin \phi \cos \psi) \\
& \ddot{z}=-g+\frac{U_{z}}{m}(\cos \phi \cos \theta),
\end{aligned}
$$

where $x, y, z$ are longitudinal, lateral, and altitude displacements and $\phi, \theta, \psi$ are roll, pitch, and yaw angles in the Earthfixed frame, respectively. $g$ is the gravity acceleration and $m$ is the mass of quadcopter UAV. $I_{x}, I_{y}, I_{z}$ are roll, pitch, and yaw inertia moments, respectively. The lift force $U_{z}$ and the aerodynamical roll $U_{\phi}$, pitch $U_{\theta}$, and heading $U_{\psi}$ moments developed by the quadcopter UAV are expressed with respect to the rotors velocities: 


$$
\begin{aligned}
& \left(\begin{array}{c}
U_{z} \\
U_{\phi} \\
U_{\theta} \\
U_{\psi}
\end{array}\right) \\
& =\left(\begin{array}{cccc}
C_{L} & C_{L} & C_{L} & C_{L} \\
0 & d C_{L} & 0 & -d C_{L} \\
-d C_{L} & 0 & d C_{L} & 0 \\
-C_{D} & C_{D} & -C_{D} & C_{D}
\end{array}\right)\left(\begin{array}{c}
\omega_{1}^{2} \\
\omega_{2}^{2} \\
\omega_{3}^{2} \\
\omega_{4}^{2}
\end{array}\right),
\end{aligned}
$$

where $C_{L}$ and $C_{D}$ are lift and drag force coefficients, respectively. $d$ is the distance between the center mass of quadcopter and the axis of the propeller. $\omega_{i}$ is the rotor $i$ velocity, $i=$ $\{1,2,3,4\}$. From (1d), (1e), and (1f), roll $(\phi)$ and pitch $(\theta)$ angles are expressed as follows:

$$
\begin{aligned}
& \phi=\arcsin \left(\frac{\ddot{x} \sin \psi-\ddot{y} \cos \psi}{\sqrt{\ddot{x}^{2}+\ddot{y}^{2}+(\ddot{z}+g)^{2}}}\right) \\
& \theta=\arctan \left(\frac{\ddot{x} \cos \psi+\ddot{y} \sin \psi}{\ddot{z}+g}\right)
\end{aligned}
$$

Using (1a)-(1f), the aerodynamical roll, pitch, and heading moments and lift force, which are induced by the reference flight trajectory, are expressed as follows:

$$
\begin{aligned}
& U_{\phi}=I_{x} \ddot{\phi} \\
& U_{\theta}=I_{y} \ddot{\theta} \\
& U_{\psi}=I_{z} \ddot{\psi} \\
& U_{z}=m \sqrt{\ddot{x}^{2}+\ddot{y}^{2}+(\ddot{z}+g)^{2}}
\end{aligned}
$$

Based on (3a) and (3b), it can be concluded that the analytical expressions of $\dot{\phi}, \dot{\theta}, \ddot{\phi}$, and $\ddot{\theta}$ are functions of the first, second, third, and fourth derivatives of the vector components $(x, y, z, \psi)$.

\section{Optimization Problem Formulation}

The optimization problem is concerned with the reference flight trajectory that satisfies waypoints constraints at specific schedule flight timetable. Since the quadcopter UAV can perform six Degrees of Freedom (DOF), the reference flight trajectory could contain six components:

$$
\mathfrak{\Im}(t)=\left(x_{d}(t), y_{d}(t), z_{d}(t), \phi_{d}(t), \theta_{d}(t), \psi_{d}(t)\right)
$$

However, the quadcopter UAV is an underactuated system. Only longitudinal, lateral, and altitude displacements $(x, y, z)$ and yaw angle $(\psi)$ can be directly controlled. Roll $(\phi)$ and pitch $(\theta)$ angles are systemically computed with respect to quadcopter dynamics. Differential flatness approach has shown interesting performance regarding the trajectory planning problem as demonstrated in $[4,5,8,12-14,16-18]$. Using the differential flatness method, the state space variables and control inputs can be defined in terms of flat output vector. Furthermore, the computation load on optimization could be reduced considerably. Equations (1a)-(1f) are differentially flat if there exists a flat output vector $\boldsymbol{\eta} \in \mathscr{R}^{m}$ in the following form [23]:

$$
\boldsymbol{\eta}=\zeta\left(\mathbf{x}, \mathbf{u}, \dot{\mathbf{u}}, \ddot{\mathbf{u}}, \ldots, \mathbf{u}^{(r)}\right)
$$

such that

$$
\begin{aligned}
& \mathbf{x}=\alpha\left(\boldsymbol{\eta}, \dot{\boldsymbol{\eta}}, \ldots, \dot{\boldsymbol{\eta}}^{(q)}\right) \\
& \mathbf{u}=\beta\left(\boldsymbol{\eta}, \dot{\boldsymbol{\eta}}, \ldots, \dot{\boldsymbol{\eta}}^{(q)}\right),
\end{aligned}
$$

where $\mathbf{x}$ and $\mathbf{u}$ are state space and control input vectors, respectively. From (1a)-(1f), (3a)-(3b), and (4a)-(4d), it can be concluded that the vector $(x, y, z, \psi)$ represents the flat output $\eta(t)$ vector. Therefore, the optimization problem can be treated from the flat output $\eta(t)$ vector and expressed as NLP problem in the following form:

$$
\begin{aligned}
\min & J(\eta(t)) \quad \text { for } t \in[0, T] \\
\text { such that: } & h(\eta(t))=0 \\
& g(\eta(t)) \leqslant 0,
\end{aligned}
$$

where (8b) and (8c) denote a set of equality and inequality constraints, respectively. $J(\eta(t))$ is the objective function.

3.1. Mission Requirements and Constraints. The considered constraints are classified as follows.

(a) Mission Constraints. The mission constraints are defined as a set of conditions as follows:

(i) The quadcopter UAV posture:

$$
\begin{aligned}
\mathfrak{I}(0) & =\mathfrak{J}_{\text {init }} \\
\mathfrak{I}\left(t_{i}\right) & =\mathfrak{J}_{i}, \quad i=1, \ldots, n_{\text {waypoint }} \\
0 & <t_{1}<t_{2}<\cdots<t_{n_{\text {waypoint }}}<T<T_{\text {aut }} \\
\mathfrak{I}(T) & =\mathfrak{I}_{\text {fin }},
\end{aligned}
$$

where $T_{\text {aut }}$ and $T$ are autonomy flight and final times, respectively. $n_{\text {waypoint }}$ is the number of waypoints.

(ii) The quadcopter UAV scheduled flight timetable:

$$
t_{i}^{*}-\delta t \leqslant t_{i} \leqslant t_{i}^{*}+\delta t
$$

where $t_{i}^{*}$ is the predefined overfly time and $\delta t$ is an allowed range of overfly time.

(iii) The quadcopter UAV velocity:

$$
\begin{aligned}
\dot{\Im}(0) & =\overrightarrow{0} \\
\dot{\mathfrak{J}}(T) & =\overrightarrow{0}
\end{aligned}
$$


(b) Workspace and Flight Envelope Constraints

(i) The workspace limits:

$$
\begin{aligned}
& \phi_{\min } \leqslant \phi_{d}(t) \leqslant \phi_{\max } \\
& \theta_{\min } \leqslant \theta_{d}(t) \leqslant \theta_{\max } \\
& \psi_{\min } \leqslant \psi_{d}(t) \leqslant \psi_{\max } \\
& x_{\min } \leqslant x_{d}(t) \leqslant x_{\max } \\
& y_{\min } \leqslant y_{d}(t) \leqslant y_{\max } \\
& z_{\min } \leqslant z_{d}(t) \leqslant z_{\max }
\end{aligned}
$$

(ii) The flight envelope limits: the flight envelope is a set of flight conditions defined by the quadcopter height and airspeed.

$$
\begin{aligned}
z_{\min } & \leqslant z_{d}(t) \leqslant z_{\max } \\
\dot{\psi}_{\min } & \leqslant \dot{\psi}_{d}(t) \leqslant \dot{\psi}_{\max } \\
0 & \leqslant V_{a} \leqslant V_{\mathrm{MO}},
\end{aligned}
$$

where $V_{\mathrm{MO}}$ is the maximum operating speed.

(c) Induced Efforts Constraints. From (2), the induced efforts can be expressed as follows:

$$
\begin{aligned}
0 & \leqslant U_{z} \leqslant 4 C_{L} \omega_{\max }^{2} \\
-d C_{L} \omega_{\max }^{2} & \leqslant U_{\phi} \leqslant d C_{L} \omega_{\max }^{2} \\
-d C_{L} \omega_{\max }^{2} & \leqslant U_{\theta} \leqslant d C_{L} \omega_{\max }^{2} \\
-2 C_{D} \omega_{\max }^{2} & \leqslant U_{\psi} \leqslant 2 C_{D} \omega_{\max }^{2}
\end{aligned}
$$

Since the quadcopter rotors are identical, $\omega_{\max }$ denotes the maximum rotor velocity.

3.2. Reference Flight Trajectory Parameterization. The reference trajectory can be defined as a composition $[3,6,15,24]$ of path and motion functions. Based on this definition, the velocity, acceleration, jerk, and snap profiles of reference flight trajectory could be adjusted to make them compliant with the assigned constraints. The reference flight trajectory $\mathfrak{I}(t)=\left(x_{d}(t), y_{d}(t), z_{d}(t), \psi_{d}(t)\right)$ is written as follows:

$$
\begin{aligned}
\mathfrak{I}(t)= & P(\lambda) \circ \lambda(t)=P(\lambda(t)) \\
\dot{\Im}(t)= & \dot{\lambda}(t) \frac{d P}{d \lambda}(\lambda(t)) \\
\ddot{\Im}(t)= & \ddot{\lambda}(t) \frac{d P}{d \lambda}(\lambda(t))+\dot{\lambda}^{2}(t) \frac{d^{2} P}{d \lambda^{2}}(\lambda(t)) \\
\dddot{\Im}(t)= & \dddot{\lambda}(t) \frac{d P}{d \lambda}(\lambda(t))+3 \dot{\lambda}(t) \ddot{\lambda}(t) \frac{d^{2} P}{d \lambda^{2}}(\lambda(t)) \\
& +\dot{\lambda}^{3}(t) \frac{d^{3} P}{d \lambda^{3}}(\lambda(t))
\end{aligned}
$$

$$
\begin{aligned}
\mathfrak{J}^{(4)}(t)= & \lambda^{(4)}(t) \frac{d P}{d \lambda}(\lambda(t)) \\
& +4 \dot{\lambda}(t) \dddot{\lambda}(t) \frac{d^{2} P}{d \lambda^{2}}(\lambda(t)) \\
& +3 \ddot{\lambda}^{2}(t) \frac{d^{2} P}{d \lambda^{2}}(\lambda(t)) \\
& +6 \dot{\lambda}^{2}(t) \ddot{\lambda}(t) \frac{d^{3} P}{d \lambda^{3}}(\lambda(t)) \\
& +\dot{\lambda}^{4}(t) \frac{d^{4} P}{d \lambda^{4}}(\lambda(t)),
\end{aligned}
$$

where the path $P(\lambda)$ is a set of time-independent configurations followed by the quadcopter UAV to fly from an initial configuration $P_{\text {init }}$ to a final one $P_{\text {fin }}$. The motion $\lambda(t)$ is a positive scalar function that defines the way in which the path is achieved with respect to the time, $\lambda \in[0,1]$. In order to construct the path $P(\lambda)$ and motion $\lambda(t)$ functions, a parametric function has to be selected. For this purpose, $\mathrm{B}$-spline function is used for its advantage to support the manipulation of the reference trajectory as functions of control points and knot vectors. Basically, B-spline function is demonstrated as follows [24]:

$$
S(u)=\sum_{i=1}^{N} c_{i} B_{i}^{p}(u)
$$

with

$$
u_{0} \leq \cdots \leq u_{j-1} \leq u_{j} \leq u_{j+1} \leq \cdots \leq u_{n_{\mathrm{knot}}},
$$

where $c_{i}$ is a control point and $N$ is a control points number. $u$ is a knot vector and $n_{\mathrm{knot}}$ is the number of knots. $B_{i}^{p}$ is a basis function of degree $p$. For this purpose, the knot vectors are selected as uniform sequences. To construct the path and motion functions, the following conditions are defined:

(a) Path Function $P(\lambda)$

(i) Posture condition: it satisfies the condition indicated in $(9 a)-(9 d)$ :

$$
\begin{aligned}
& P(\lambda=0)=P_{\text {init }} \\
& P\left(\lambda_{i}\right)=P_{i}, \quad i=1, \ldots, n_{\text {waypoint }} \\
& P(\lambda=1)=P_{\text {fin }}
\end{aligned}
$$

(ii) Velocity condition: it ensures the condition of (11a) and (11b):

$$
\begin{aligned}
& \dot{P}(\lambda=0)=\overrightarrow{0}, \\
& \dot{P}(\lambda=1)=\overrightarrow{0}
\end{aligned}
$$

(c) Motion Function $\lambda(t)$

(i) The first derivative of $\lambda(t)$ :

$$
\frac{d \lambda}{d t} \geqslant 0, \quad \forall t \in[0, T]
$$




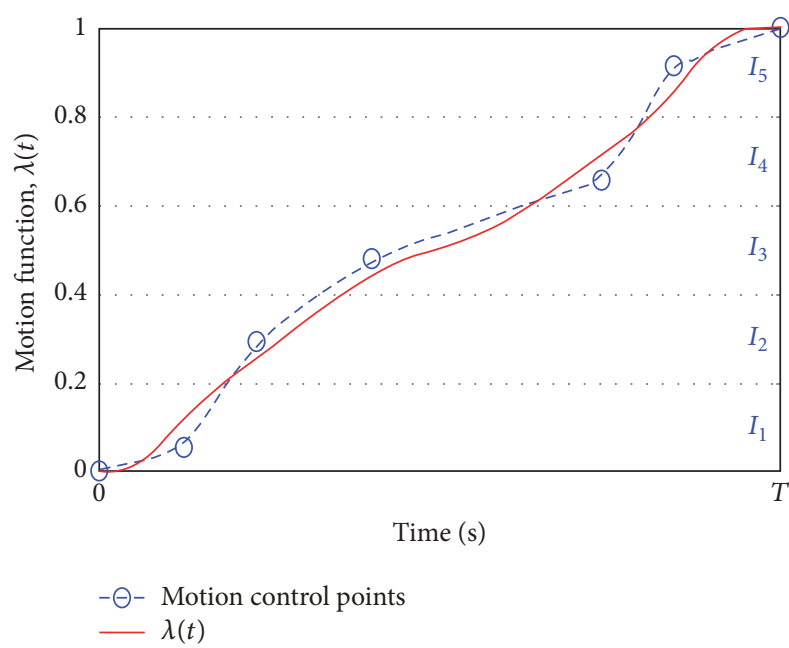

(a)

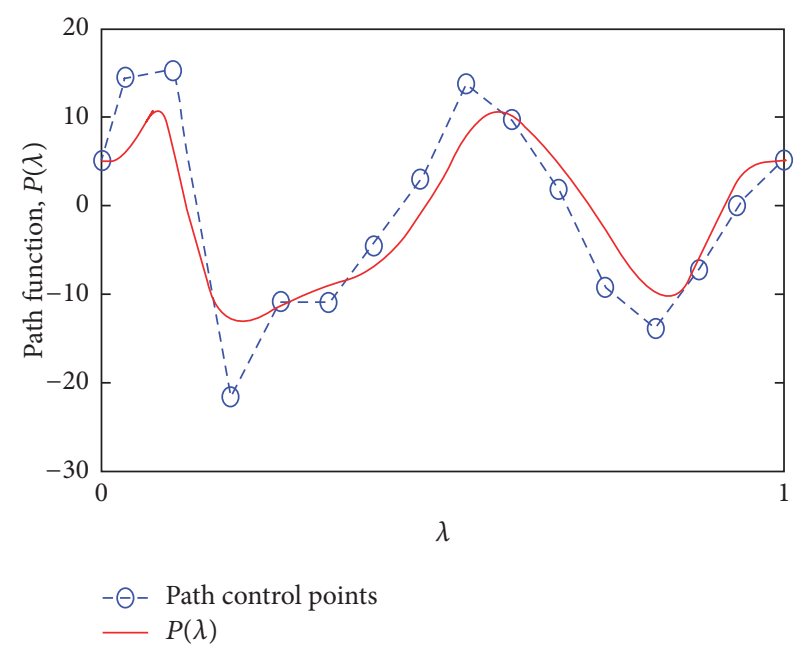

(b)

FIGURE 1: An illustration example for (a) motion $\lambda(t)$ and (b) path $P(\lambda)$ functions, respectively.

(ii) The motion must satisfy the following conditions:

$$
\begin{aligned}
& \lambda(0)=0 \\
& \dot{\lambda}(0)=0 \\
& \ddot{\lambda}(0)=0 \\
& \dddot{\lambda}(0)=0 \\
& \lambda(T)=1 \\
& \dot{\lambda}(T)=0 \\
& \ddot{\lambda}(T)=0 \\
& \dddot{\lambda}(T)=0
\end{aligned}
$$

(iii) The overfly-time conditions:

$$
\lambda\left(t_{i}\right)=\lambda_{i}, \quad i=1, \ldots, n_{\text {waypoint }}
$$

From (3a)-(3b) and (15a)-(15e), the continuity of the second-order derivatives of the desired roll $\phi_{d}(t)$ and pitch $\theta_{d}(t)$ angles depends on the continuity of the fourth derivative of the path and motion functions. Therefore, the parametric function of $P(\lambda)$ is modeled as a quintic B-spline function using $\left(N_{p}+2\right)$ control points number, where $N_{p} \geqslant 5$. The $\left(N_{p}\right)$ control points number represents a set of path control points that will be manipulated by the proposed optimization method. The (2) control points number represents the fixed control points that satisfy the condition expressed in (18a) and (18c). The parametric function of $\lambda(t)$ is modeled as a quintic B-spline function using $\left(N_{m}+8\right)$ control points number. The $\left(N_{m}\right)$ control points number is a set of motion control points and the (8) control points number is a set of fixed control points that meet conditions defined by (11a)-(11b) and (2la)-(21h). In addition, the motion control points' vector is uniformly distributed along the time scale, guarantying that each motion control point belongs to an increasing bounded interval to satisfy the condition of (20). Figure 1 illustrates an example for path and monotonically increasing motion functions, respectively.

\section{Performance Criterion}

For rotor-craft systems, the electrical power is converted to mechanical power in the form of thrust. The developed mechanical power could be described as the product of the torque $\tau_{i}$ and angular velocity $\omega_{i}$. Therefore, the power generated from each rotor $i$ could be computed as follows:

$$
P_{i}=\tau_{i} \omega_{i}=\left(C_{D} \omega_{i}^{2}\right) \omega_{i}
$$

By using the first term $C_{D} \omega_{i}^{2}$ in (23), the proposed cost function is expressed as a function of rotor velocities and yaw dynamics:

$$
\begin{aligned}
J & =\int_{0}^{T}\left[\left(\sum_{i=1}^{4} C_{D} \omega_{i}^{2}\right)^{2}+\ddot{\psi}^{2}\right] d t \\
& =\int_{0}^{T}\left[C_{D}^{2}\left(\omega_{1}^{2}+\omega_{2}^{2}+\omega_{3}^{2}+\omega_{4}^{2}\right)^{2}+\ddot{\psi}^{2}\right] d t \\
& =\int_{0}^{T}\left[\beta\left(\ddot{x}^{2}+\ddot{y}^{2}+(\ddot{z}+g)^{2}\right)+\ddot{\psi}^{2}\right] d t
\end{aligned}
$$

with

$$
\beta=\left(C_{D} \frac{m}{C_{L}}\right)^{2}
$$

where the term $\ddot{\psi}^{2}$ is used to minimize the induced aerodynamical heading moment and the second term $\beta\left(\ddot{x}^{2}+\ddot{y}^{2}+(\ddot{z}+\right.$ 


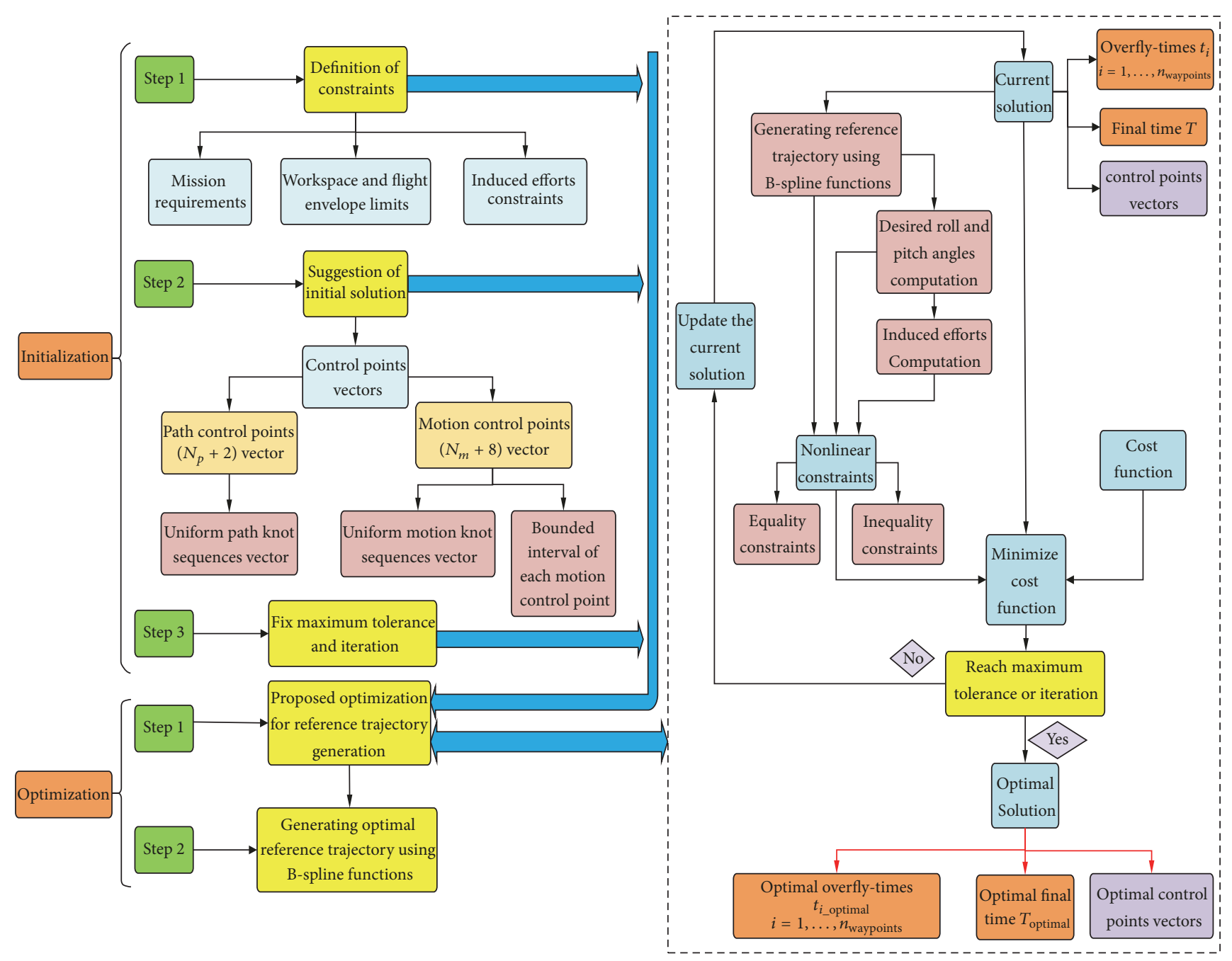

FIGURE 2: Proposed optimization method procedure.

$g)^{2}$ ) is introduced in the proposed cost function to minimize the induced efforts and consumed energy. This yields

$$
\begin{aligned}
J= & \int_{0}^{1}\left[\beta\left(\left(\frac{d^{2} z_{1}}{d \lambda^{2}}\right)^{2}+\left(\frac{d^{2} z_{2}}{d \lambda^{2}}\right)^{2}+\left(\frac{d^{2} z_{3}}{d \lambda^{2}}+g\right)^{2}\right)\right. \\
& \left.+\left(\frac{d^{2} z_{4}}{d \lambda^{2}}\right)^{2}\right] d \lambda
\end{aligned}
$$

The synoptic procedure of optimization method is shown in Figure 2. For fixed scheduled flight timetable problem, only the positions of the control points' vectors are manipulated. The final solution will be the optimal control points' vectors. For this problem, the overfly-time constraints defined should be selected properly. For the requirements of the optimal overfly-times schedule, the overfly times are calculated by the proposed optimization problem with respect to the constraints of interest and performance criteria.

\section{Numerical Simulations}

5.1. Flight Trajectory 1. Let us consider the following configuration in Table 1. All simulations are done using $N_{m}=$
TABLE 1: Waypoints configuration with their scheduled flight timetable.

\begin{tabular}{lccccc}
\hline Waypoint & $t(\mathrm{~s})$ & $x(\mathrm{~m})$ & $y(\mathrm{~m})$ & $z(\mathrm{~m})$ & $\psi(\mathrm{rad})$ \\
\hline Initial & 0 & 5 & -5 & 0 & 0 \\
1 & 10 & 10 & 0 & 20 & -0.1745 \\
2 & 25 & 0 & 15.708 & 20 & 0.3491 \\
3 & 43 & -10 & 0 & 20 & 0.5236 \\
4 & 68 & 0 & -15.708 & 20 & 0.1745 \\
5 & 80 & 10 & 0 & 20 & 0 \\
Final & 95 & 5 & 5 & 0 & 0
\end{tabular}

12 and $N_{p}=14$. The constraints are listed in Table 3. The real parameters identified in [20] are used to test the proposed optimization method (see Table 2). A numerical solver in Matlab [25] via "fmincon" based upon Sequential Quadratic Programming (SQP) is used to solve the optimization method. To demonstrate the effectiveness of proposed cost function in (26), different cost functions defined in $[15,17]$ are used. Figure 3 displays the $2 \mathrm{D}$ and 3D reference flight trajectory with respect to the requirements of Table 1. 


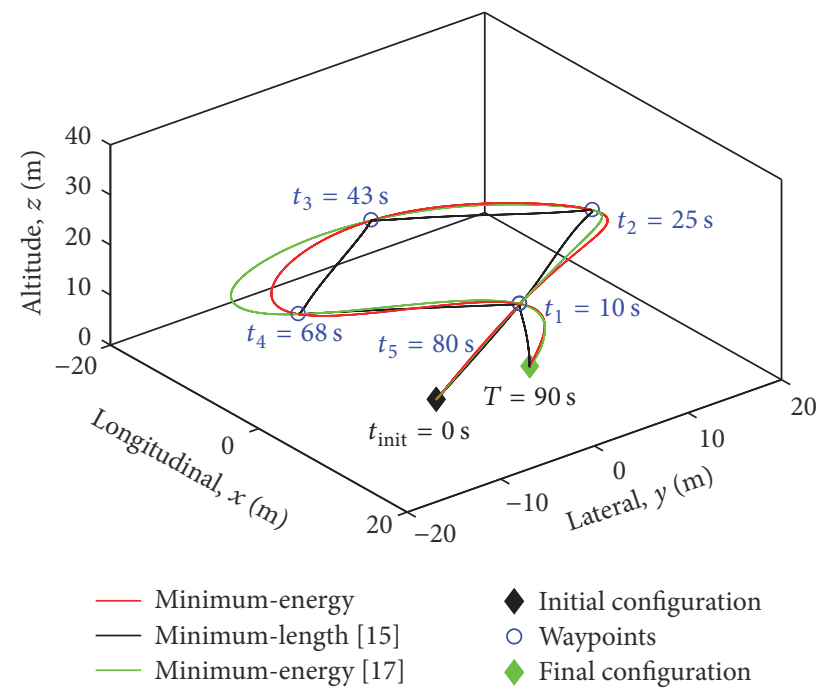

(a)

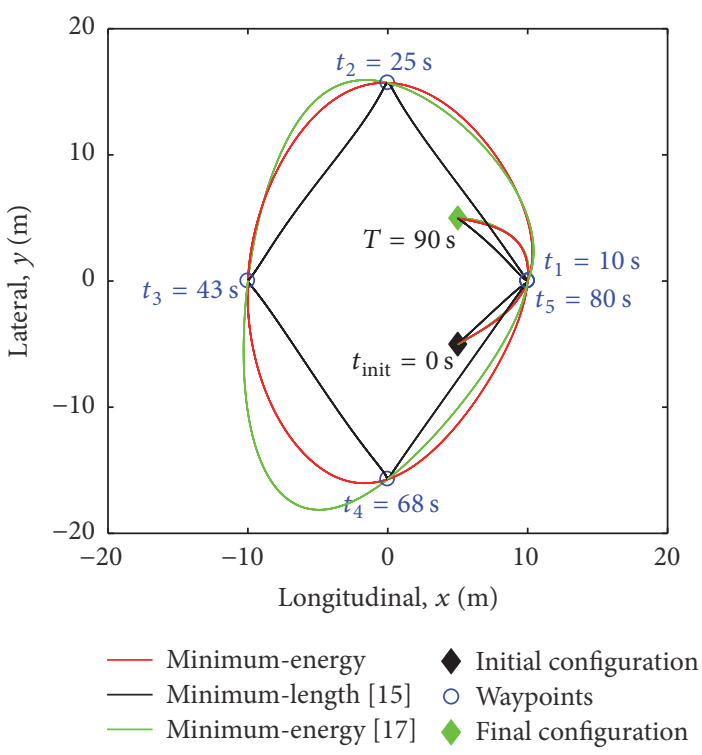

(b)

Figure 3: Optimal 3D and 2D reference flight trajectory.

TABLE 2: Quadcopter UAV parameters [20].

\begin{tabular}{lc}
\hline Parameter & Value \\
\hline$I_{x}$ & $0.0059 \mathrm{~kg} \cdot \mathrm{m}^{2}$ \\
$I_{y}$ & $0.0059 \mathrm{~kg} \cdot \mathrm{m}^{2}$ \\
$I_{z}$ & $0.0107 \mathrm{~kg} \cdot \mathrm{m}^{2}$ \\
$m$ & $0.694 \mathrm{~kg}$ \\
$C_{D}$ & $1.18 \times 10^{-7} \mathrm{~N} \cdot \mathrm{m} / \mathrm{rad} / \mathrm{s}$ \\
$C_{L}$ & $3.8162 \times 10^{-6} \mathrm{~N} / \mathrm{rad} / \mathrm{s}$ \\
$d$ & $0.18 \mathrm{~m}$ \\
\hline
\end{tabular}

TABLE 3: Considered constraints.

\begin{tabular}{lcc}
\hline $\begin{array}{l}\text { Constraint } \\
\text { types }\end{array}$ & Constraints & Constraint ranges \\
\hline & Longitudinal & $-30 \leqslant x \leqslant 30$ \\
& displacement $(\mathrm{m})$ & $-40 \leqslant y \leqslant 40$ \\
Woteral displacement $(\mathrm{m})$ & $0 \leqslant z \leqslant 120$ \\
& Altitude $(\mathrm{m})$ & $-0.5236 \leqslant \phi \leqslant 0.5236$ \\
& Roll angle $(\mathrm{rad})$ & $-0.6109 \leqslant \theta \leqslant 0.6109$ \\
& Pitch angle $(\mathrm{rad})$ & $-0.7854 \leqslant \psi \leqslant 0.7854$ \\
Flight envelope & Yaw angle $(\mathrm{rad})$ & $0 \leqslant 120$ \\
& Yaw rate $(\mathrm{rad} / \mathrm{s})$ & $-1.7454 \leqslant \dot{\psi} \leqslant 1.7454$ \\
Actuators & Airspeed $(\mathrm{m} / \mathrm{s})$ & $0 \leqslant V_{a} \leqslant 8$ \\
\hline & Maximum $\mathrm{rotor}$ & 0 \\
& velocity $(\mathrm{rad} / \mathrm{s})$ & \\
\hline
\end{tabular}

It could be noted that the proposed algorithm can deal with different cost functions. Moreover, the smoothness of the generated reference flight trajectory depends on the considered cost functions. Figure 4 shows the generated
TABLE 4: Comparison results of trajectory length and fitness value.

\begin{tabular}{lccc}
\hline & Cost function type & Trajectory length $(\mathrm{m})$ & Fitness value \\
\hline 1 & Minimum-energy & 129.16 & $1.1174 \times 10^{3}$ \\
2 & Minimum-length [15] & 117.05 & $1.3609 \times 10^{4}$ \\
3 & Minimum-energy [17] & 136.25 & $1.2241 \times 10^{4}$ \\
\hline
\end{tabular}

longitudinal, lateral, and altitude displacements in addition to yaw angle. Figures 6 and 7 illustrate the induced efforts developed by each cost function. It could be seen from the proposed cost function in (26) that the mean values of the induced efforts are considerably less than those related to the cost functions in $[15,17]$. Figure 5 displays the results given by each cost function in regards of trajectory length and consumed energy. The fitness value developed by the proposed cost function, which reflects consumed energy, is less important than other cost functions. The proposed cost function shows an interesting performance in terms of trajectory length, induced efforts, consumed energy, and smoothness, as demonstrated in Tables 4 and 5.

5.2. Flight Trajectory 2. Let us consider the configuration in Table 6. Simulations are done by considering three scenarios. In the first one, the waypoints overfly times are fixed. In the second one, the waypoints overfly times are freely computed by the proposed optimization method. However, in the last case, an allowed range of overfly time $(\delta t=5 \mathrm{~s})$ is used.

Figure 8 displays the 3D and 2D generated reference flight trajectory for the three scenarios. Even if the imposed waypoints overfly times defined in Table 6 are fixed without any prior knowledge on optimality aspect, the proposed optimization method will show its ability to generate the reference trajectory of desired configuration despite its weakness in 


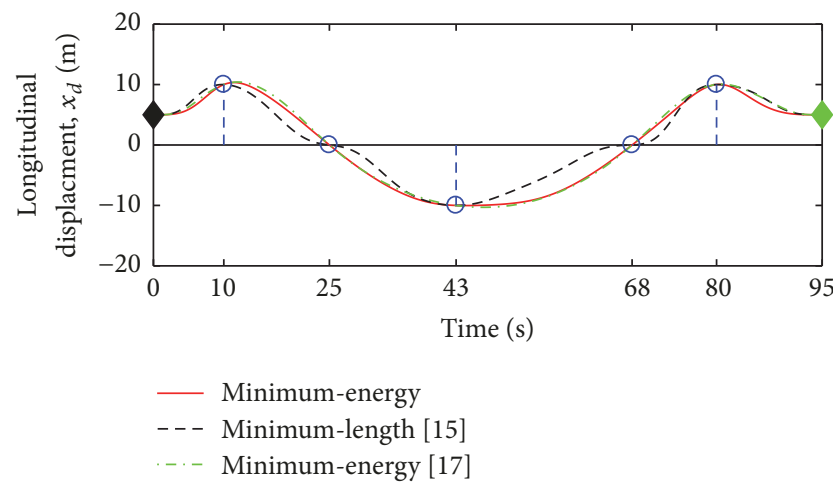

(a)

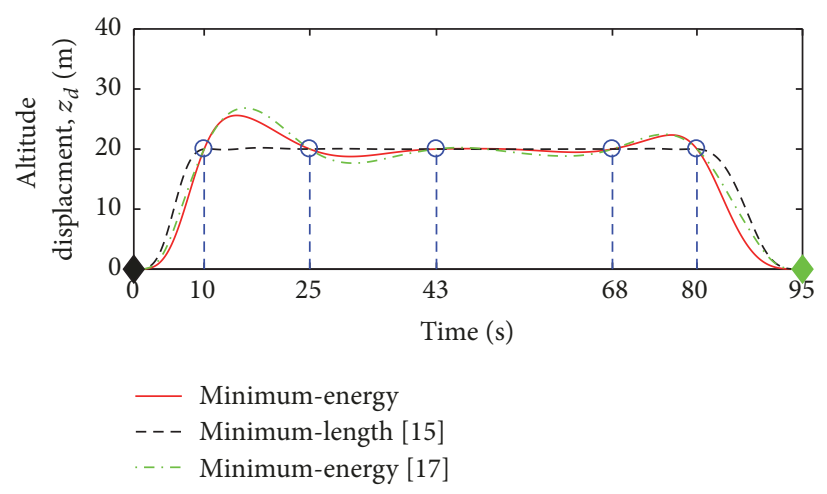

(c)

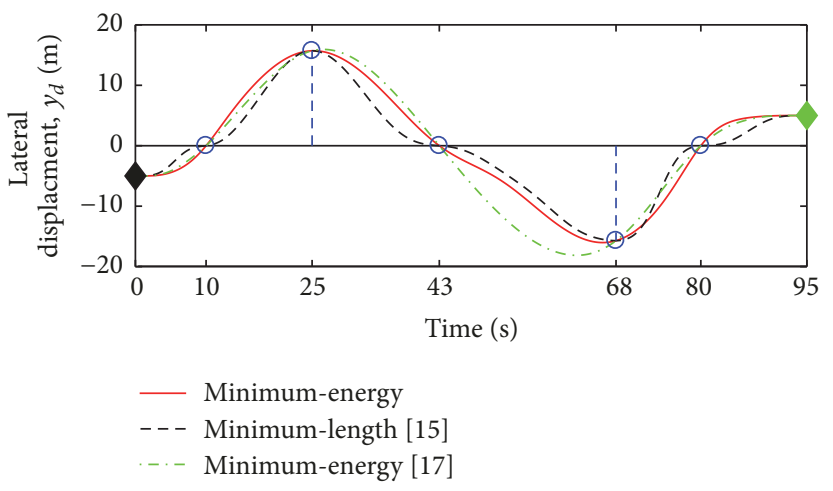

(b)

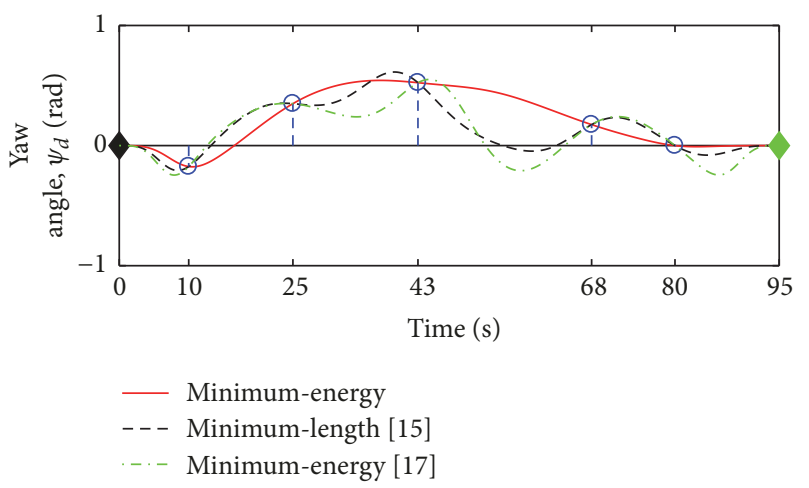

(d)

FIGURE 4: Desired (a) longitudinal, (b) lateral, and (c) altitude displacements and (d) heading angle, respectively.

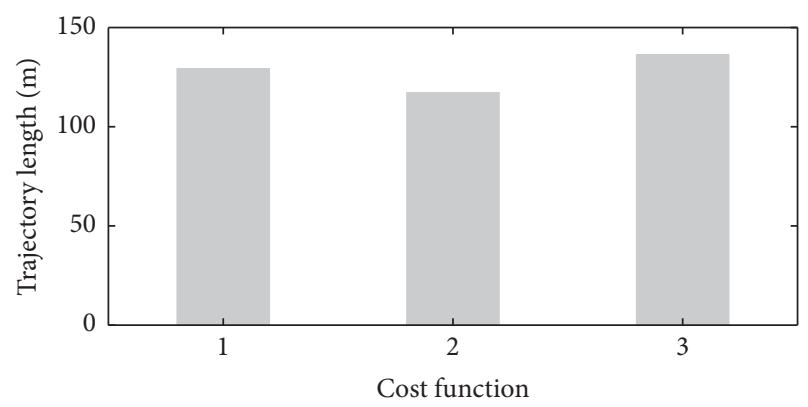

(a)

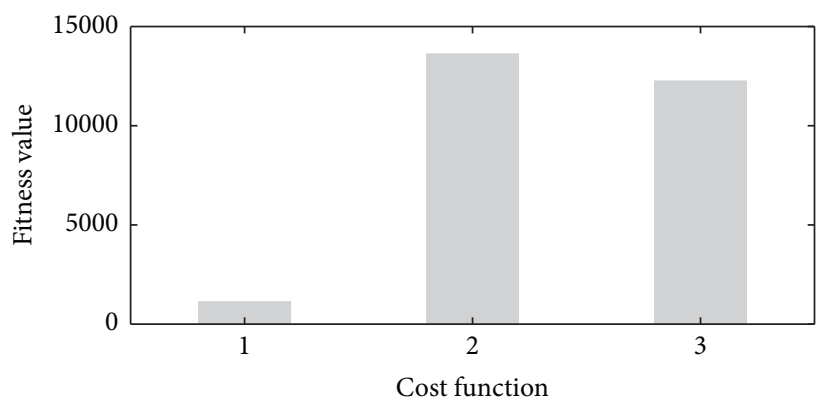

(b)

Figure 5: Performance criterion: (a) trajectory length and (b) fitness value, respectively.

terms of induced efforts, length trajectory, and consumed energy, as demonstrated in Figures 9 and 11. Table 8 shows the optimal overfly-times schedule computed in the second and third scenarios.

Figures 10 and 11 illustrate the induced lift force, aerodynamical moments, and their mean values computations with respect to each scenario. Based on the proposed optimization problem, the overfly times are computed optimally and consequently; the induced efforts, trajectory length, and consumed energy could be considerably reduced. Tables 7 and 9 show this aspect.

5.3. Flight Trajectory 3. Let us consider the same configuration in Table 6. In this study, different $\delta t$ is used and $T_{\text {aut }}=$
$20 \mathrm{~min}$ is fixed. Figure 12 displays the $3 \mathrm{D}$ and $2 \mathrm{D}$ generated reference flight trajectory and Table 10 shows the optimal overfly-times schedule computed for each selected $\delta t$. Figures 13,14 , and 15 display the trajectory length, consumed energy, and induced efforts developed by selecting $\delta t$. Tables 11 and 12 display the performance criteria assessment. It can be seen that while $\delta t$ increased, the performance criteria showed enhancing results.

\section{Conclusion}

An optimization method for reference flight trajectory generation has been proposed, which allows the generation of feasible and realistic flight plan for quadcopter UAV and manages 


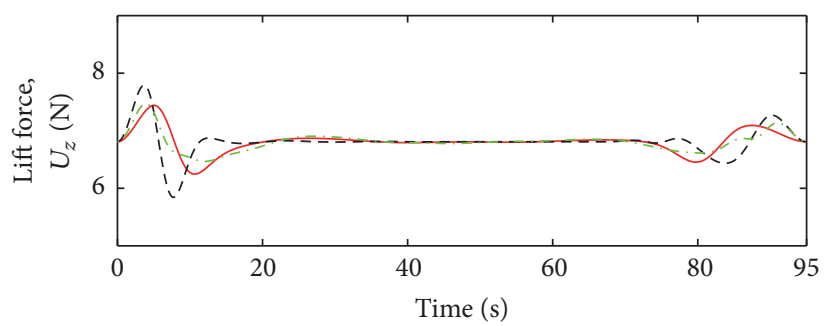

— Minimum-energy

- - - Minimum-length [15]

- . - Minimum-energy [17]

(a)

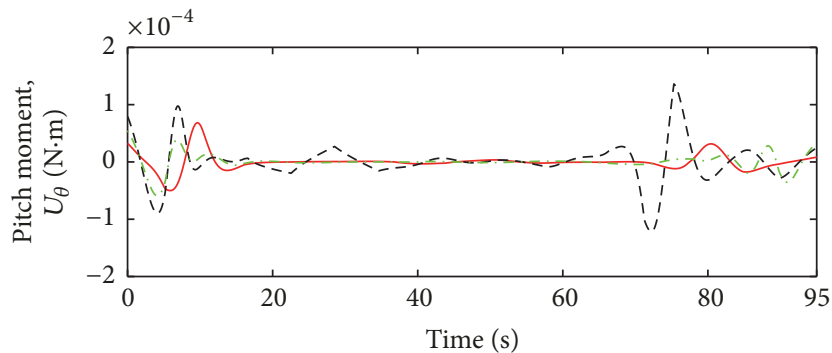

— Minimum-energy

- - - Minimum-length [15]

Minimum-energy [17]

(c)

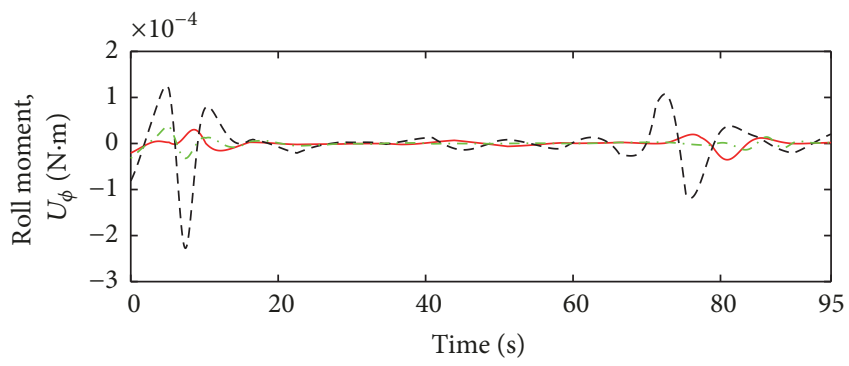

- Minimum-energy

- - Minimum-length [15]

- . - Minimum-energy [17]

(b)

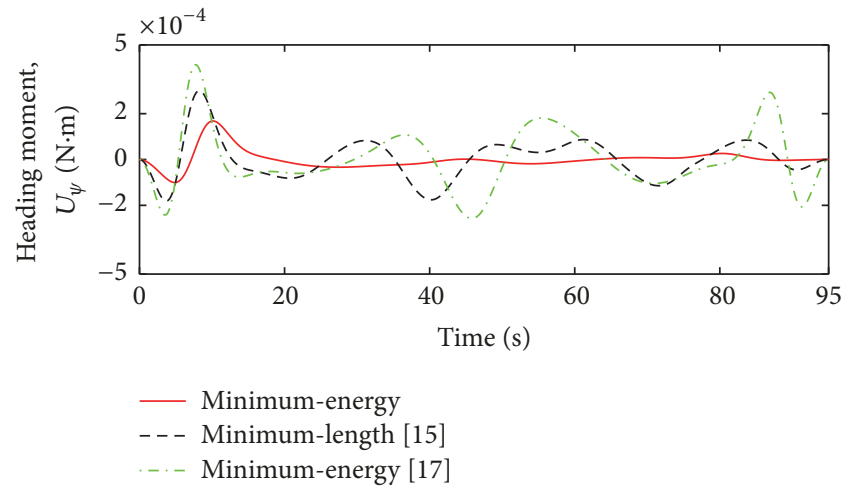

(d)

FIGURE 6: Induced efforts: (a) lift force and aerodynamical (b) roll and (c) pitch and (d) heading moments, respectively.

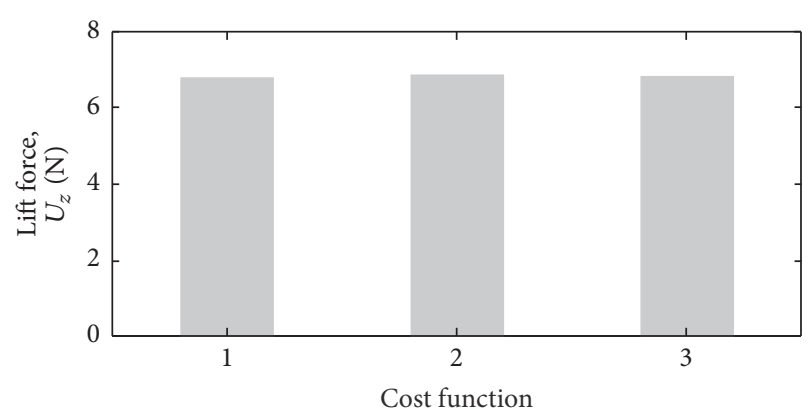

(a)

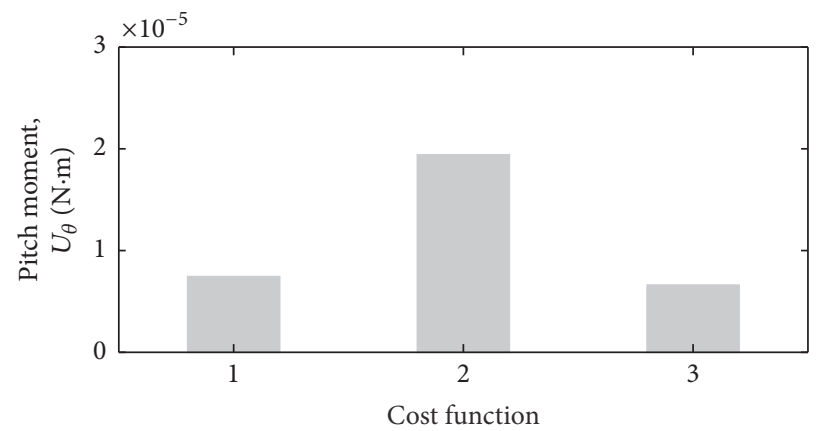

(c)

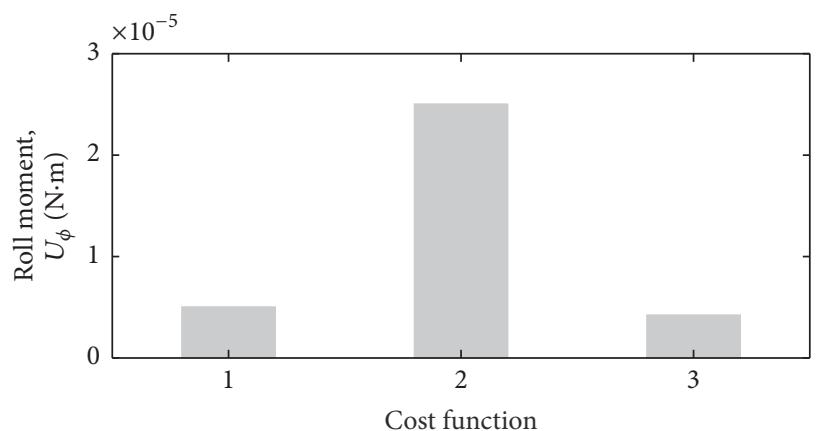

(b)

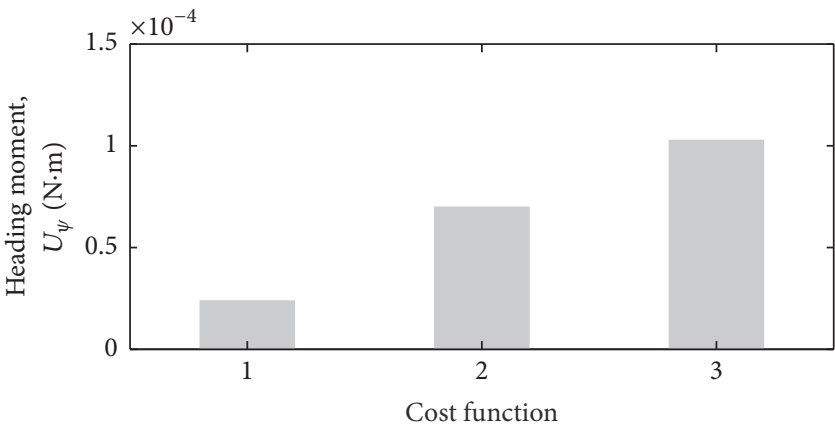

(d)

Figure 7: Mean values of (a) lift force and aerodynamical (b) roll and (c) pitch and (d) heading moments, respectively. 
TABLE 5: Comparison results of induced efforts.

\begin{tabular}{|c|c|c|c|c|c|}
\hline & Cost function type & $\begin{array}{l}\text { Lift force } \\
(\mathrm{N})\end{array}$ & $\begin{array}{l}\text { Roll moment } \\
(\mathrm{N} \cdot \mathrm{m})\end{array}$ & $\begin{array}{c}\text { Pitch moment } \\
(\mathrm{N} \cdot \mathrm{m})\end{array}$ & $\begin{array}{l}\text { Heading moment } \\
(\mathrm{N} \cdot \mathrm{m})\end{array}$ \\
\hline 1 & Minimum-energy & 6.8092 & $5.0493 \times 10^{-6}$ & $7.4343 \times 10^{-6}$ & $2.3455 \times 10^{-5}$ \\
\hline 2 & Minimum-length [15] & 6.811 & $2.4896 \times 10^{-5}$ & $1.9401 \times 10^{-5}$ & $6.9616 \times 10^{-5}$ \\
\hline 3 & Minimum-energy [17] & 6.8089 & $4.1299 \times 10^{-6}$ & $6.5995 \times 10^{-6}$ & $1.0246 \times 10^{-4}$ \\
\hline
\end{tabular}

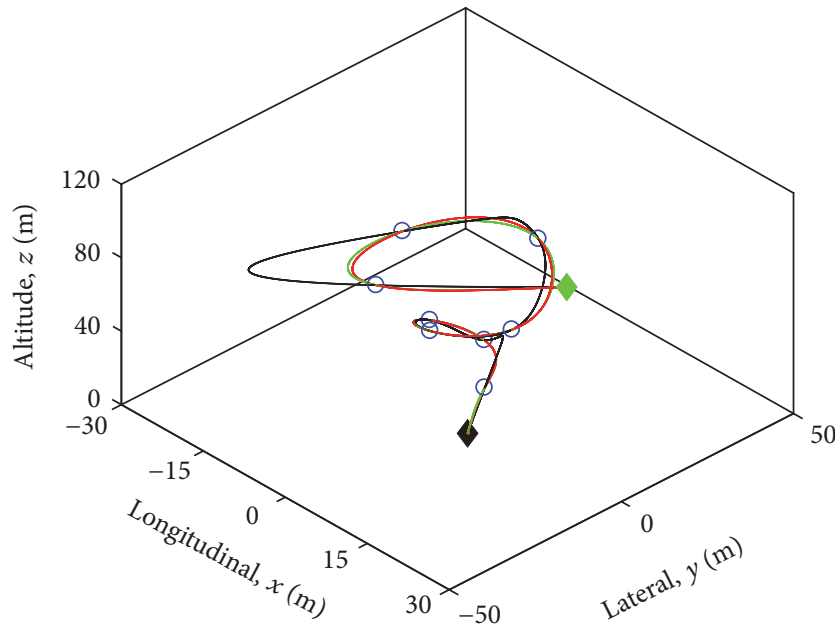

- Scenario 1

- Scenario 2

- Scenario 3

(a)

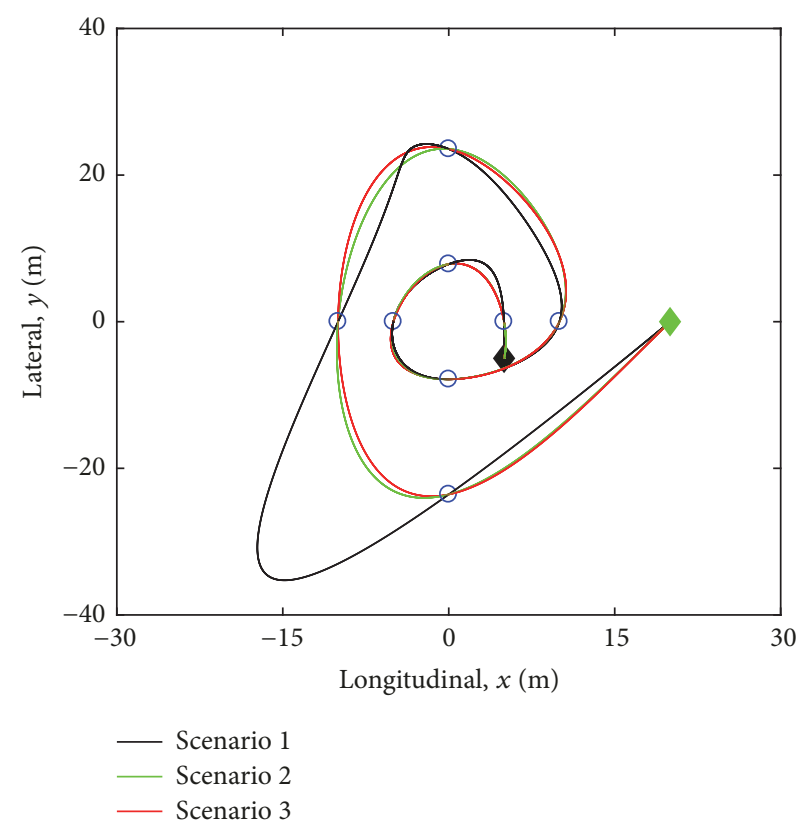

(b)

FIGURE 8: Optimal 3D and 2D reference flight trajectory.

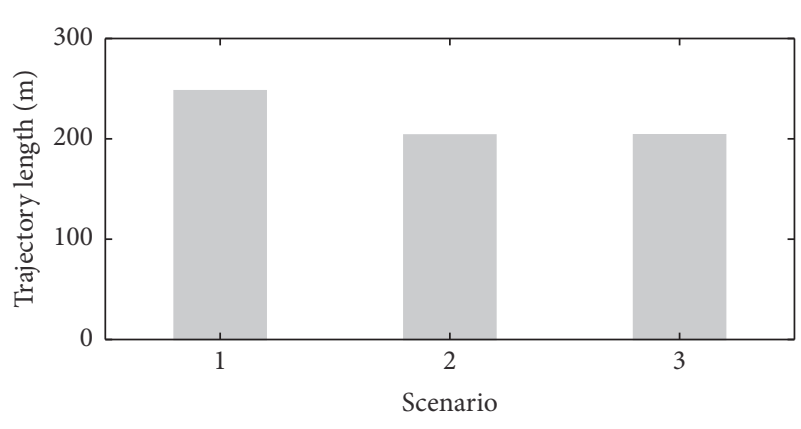

(a)

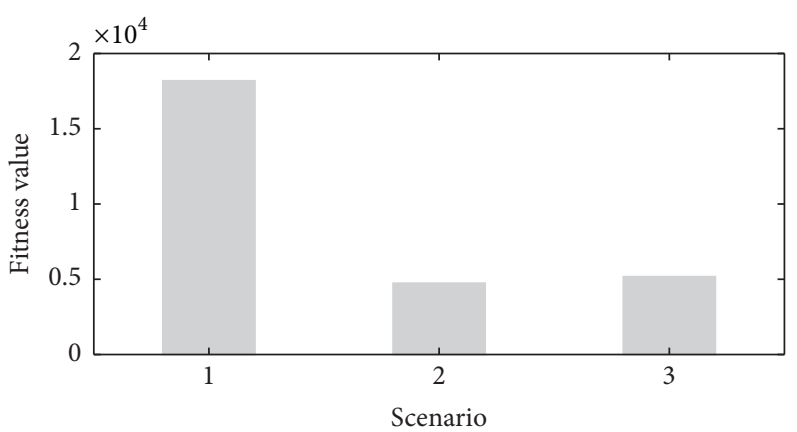

(b)

Figure 9: Performance criterion: (a) trajectory length and (b) fitness value, respectively.

adequately the scheduled flight timetable of waypoints. In this paper, the flight trajectory generation problem was treated as NLP problem and solved via a nonlinear constrained optimization technique. The reference flight trajectory was defined as a composition of path and motion functions. Both of them were generated by using B-spline functions. Based on differential flatness approach, the quadcopter UAV dynamical constraints were checked instantaneously by computing the induced aerodynamical roll, pitch, and heading moments and lift force, respectively. The optimal reference trajectory was reached by manipulating the control points' vectors of Bspline functions while taking into account the quadcopter mission requirements, dynamical constraints, flight envelope, and workspace limits. The mission requirements were defined as a series of waypoints with their respective scheduled flight timetable. A minimum-energy cost function has been proposed to minimize the consumed energy and induced efforts along the path. For requirement of optimal overfly-times schedule, the overfly times were computed in an optimal way regarding the defined constraints and performance criteria. 


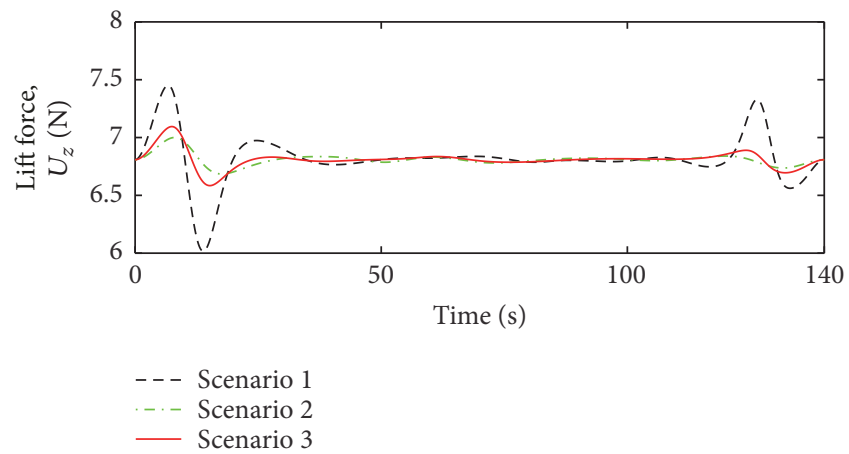

(a)

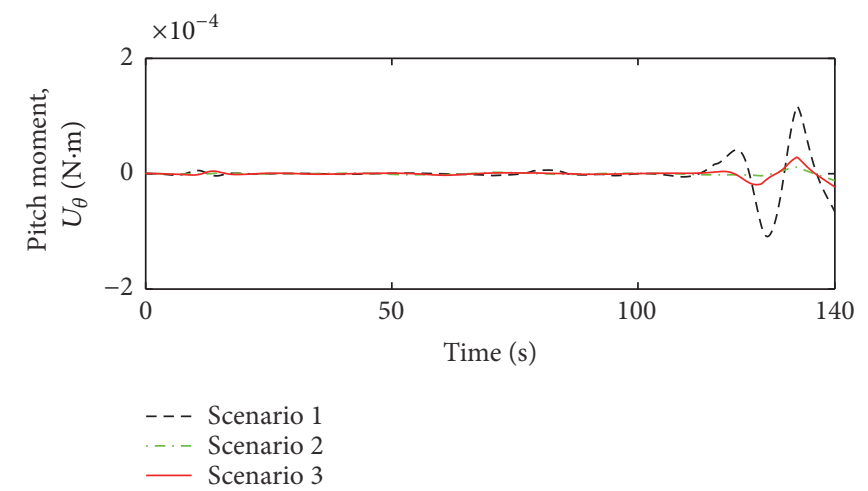

(c)

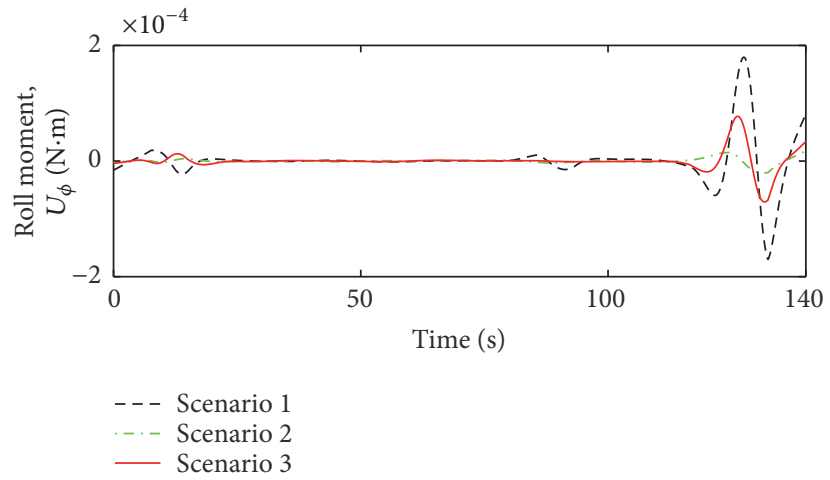

(b)

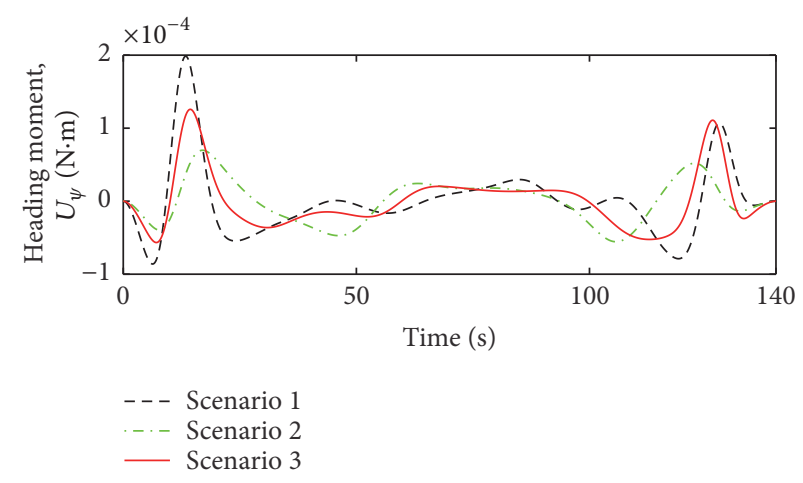

(d)

FIGURE 10: Induced efforts: (a) lift force and aerodynamical (b) roll and (c) pitch and (d) heading moments, respectively.

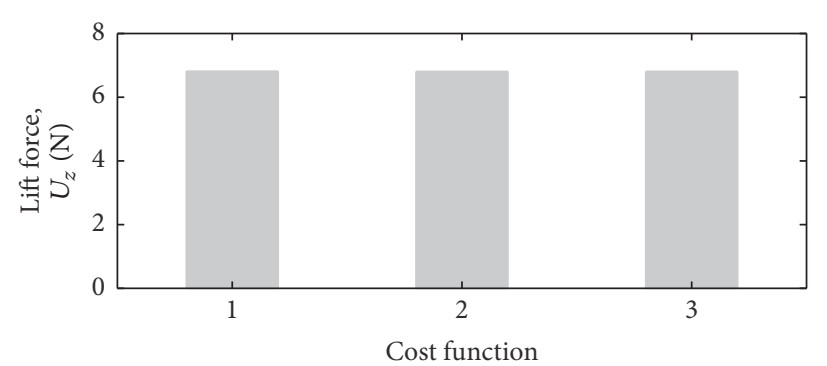

(a)

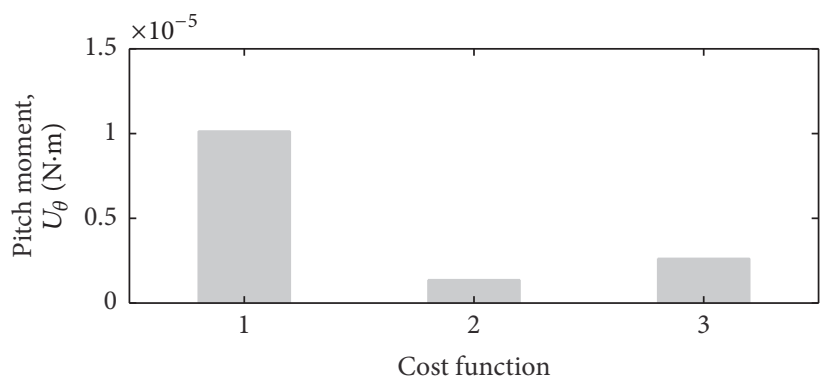

(c)

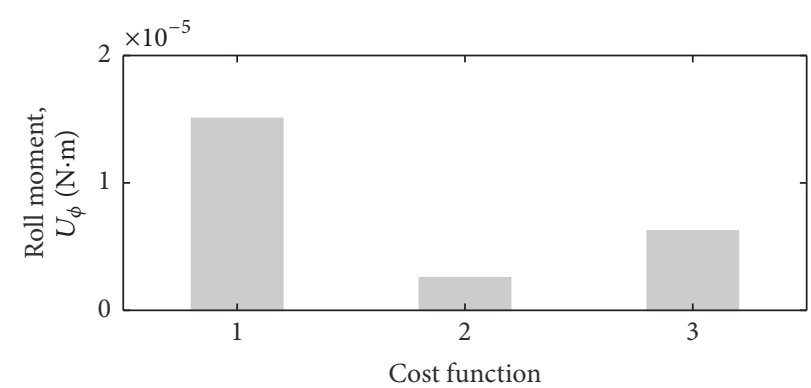

(b)

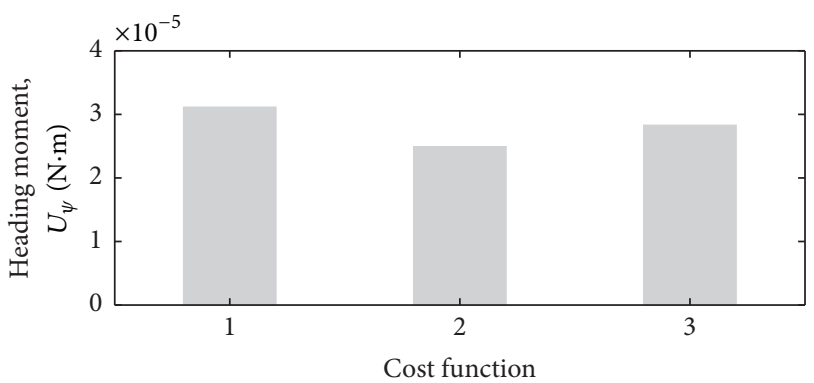

(d)

FIGURE 11: Mean values of (a) lift force and aerodynamical (b) roll and (c) pitch and (d) heading moments, respectively. 


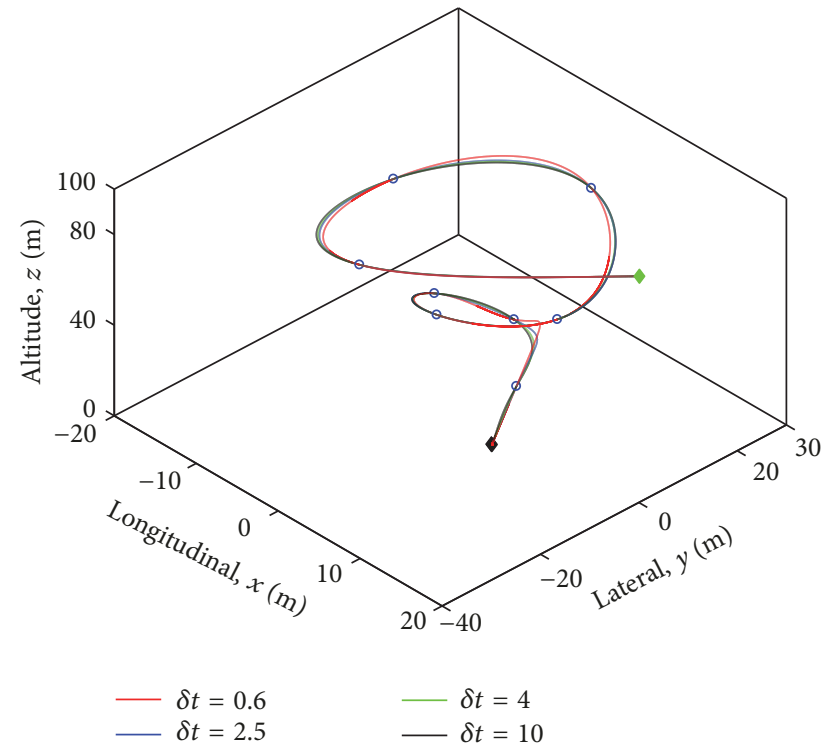

(a)

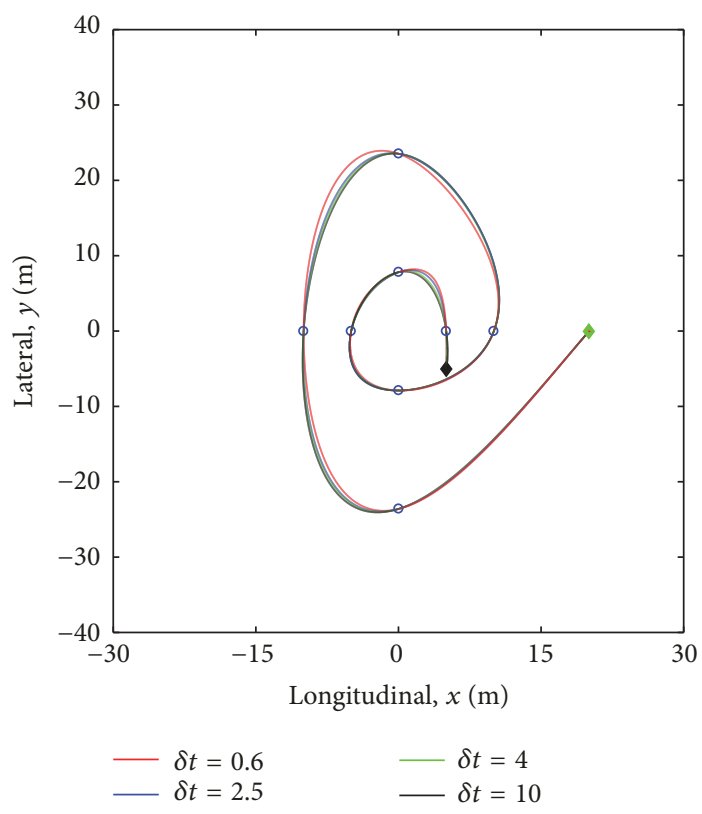

(b)

FIGURE 12: Optimal 3D and 2D reference flight trajectory.

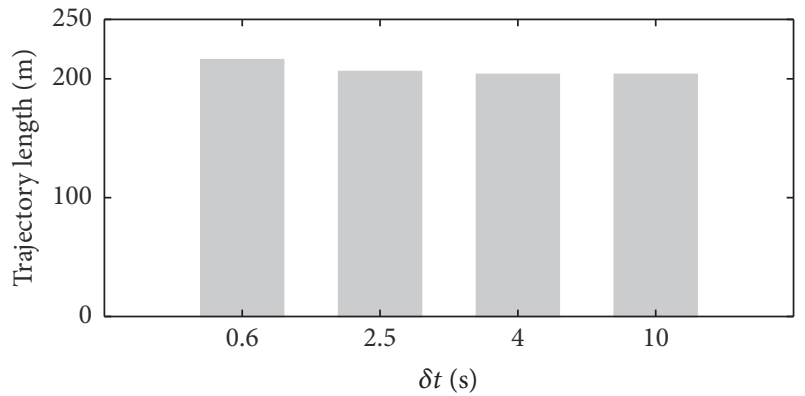

(a)

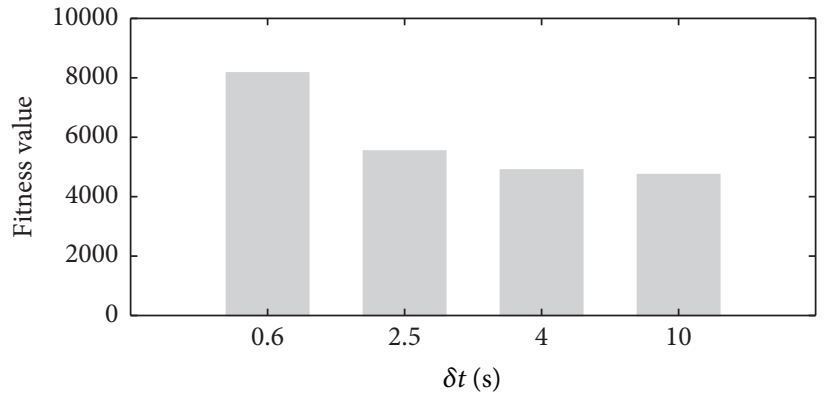

(b)

FIGURE 13: Performance criterion: (a) trajectory length and (b) fitness value, respectively.

TABLE 6: Waypoints configuration with their scheduled flight timetable.

\begin{tabular}{lccccc}
\hline Waypoint & $t(\mathrm{~s})$ & $x(\mathrm{~m})$ & $y(\mathrm{~m})$ & $z(\mathrm{~m})$ & $\psi(\mathrm{rad})$ \\
\hline Initial & 0 & 5 & -5 & 0 & 0 \\
1 & 10 & 5 & 0 & 20 & -0.1745 \\
2 & 25 & 0 & 7.854 & 30 & 0.3491 \\
3 & 43 & -5 & 0 & 40 & 0.5236 \\
4 & 68 & 0 & -7.854 & 50 & 0.1745 \\
5 & 80 & 10 & 0 & 60 & 0 \\
6 & 95 & 0 & 23.5619 & 70 & 0.1745 \\
7 & 118 & -10 & 0 & 80 & 0.4363 \\
8 & 130 & 0 & -23.5619 & 90 & 0 \\
Final & 140 & 20 & 0 & 100 & 0 \\
\hline
\end{tabular}

Numerical simulations in this study have shown the feasibility and effectiveness of the proposed optimization method to
TABLE 7: Comparison results of trajectory length and fitness value.

\begin{tabular}{lcc}
\hline Scenario & Trajectory length $(\mathrm{m})$ & Fitness value \\
\hline 1 & 247.85 & $1.8187 \times 10^{4}$ \\
2 & 203.77 & $4.7394 \times 10^{3}$ \\
3 & 203.95 & $5.1734 \times 10^{3}$ \\
\hline
\end{tabular}

generate an optimal reference flight trajectory in terms of trajectory length, induced efforts, consumed energy, and smoothness. Moreover, it shows its ability to manage the scheduled flight timetable of waypoints.

\section{Conflicts of Interest}

The authors declare that there are no conflicts of interest regarding the publication of this paper. 


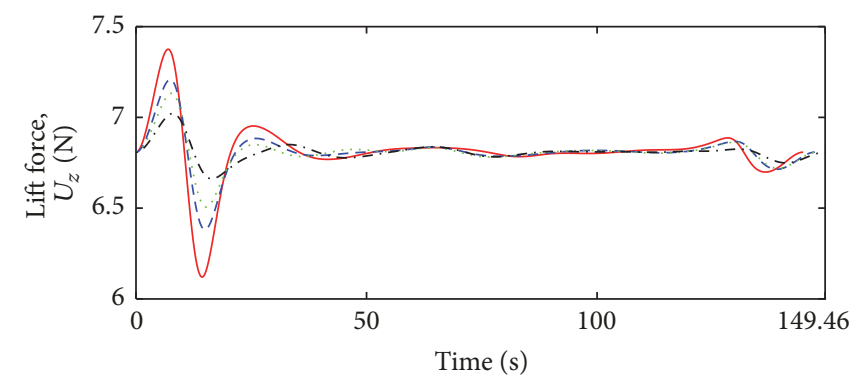

$$
\begin{aligned}
& -\delta t=0.6 \\
& ---\delta t=2.5 \\
& \text { … } \delta t=4 \\
& \text {-..- } \delta t=10
\end{aligned}
$$

(a)

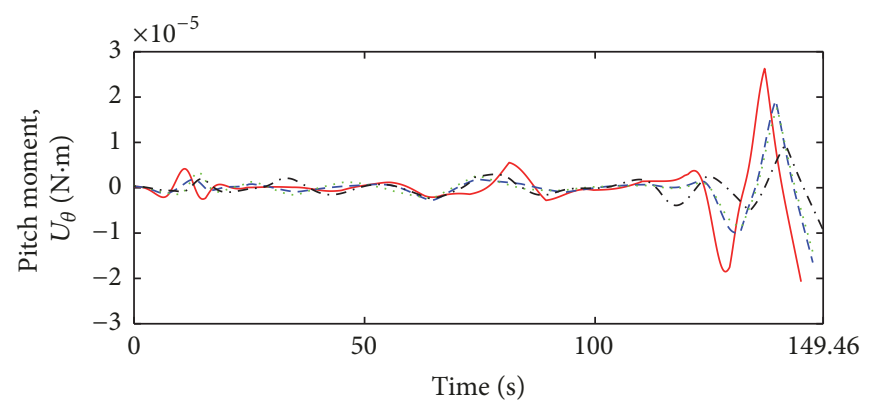

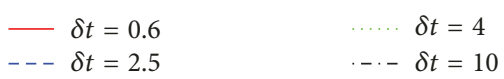

(c)

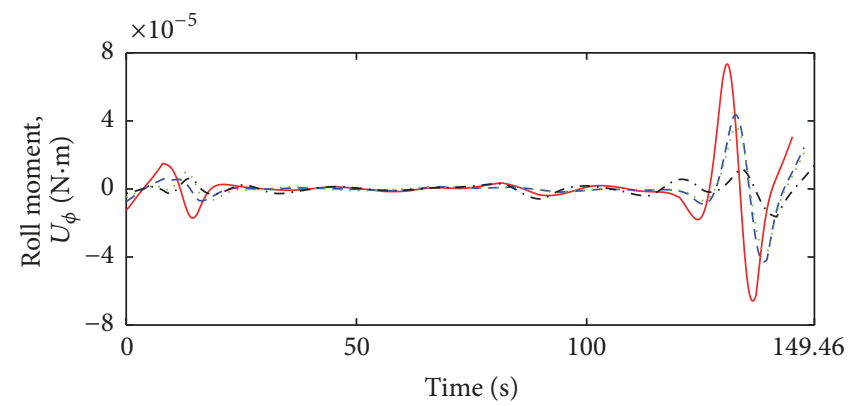

$-\delta t=0.6$

$\cdots . . . \delta t=4$

$---\delta t=2.5$

$\cdots \delta t=10$

(b)

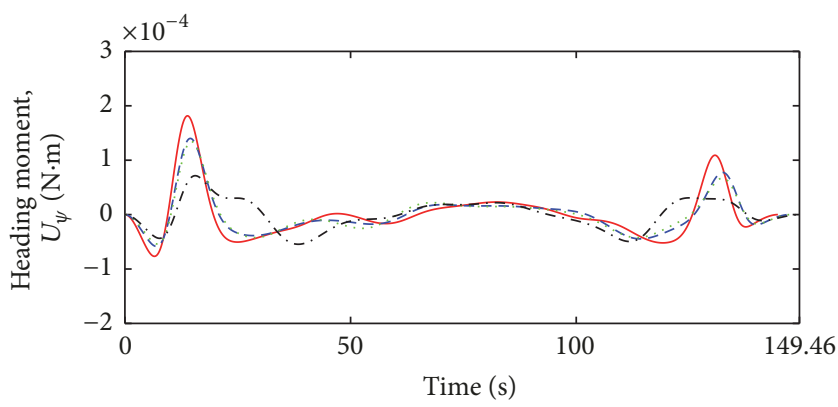

$$
\begin{aligned}
& \begin{aligned}
-\delta t & =0.6 \\
--\delta t & =2.5
\end{aligned} \\
& \begin{array}{l}
\ldots \delta t=4 \\
--\delta t=10
\end{array}
\end{aligned}
$$

(d)

FIGURE 14: Induced efforts: (a) lift force and aerodynamical (b) roll and (c) pitch and (d) heading moments, respectively.

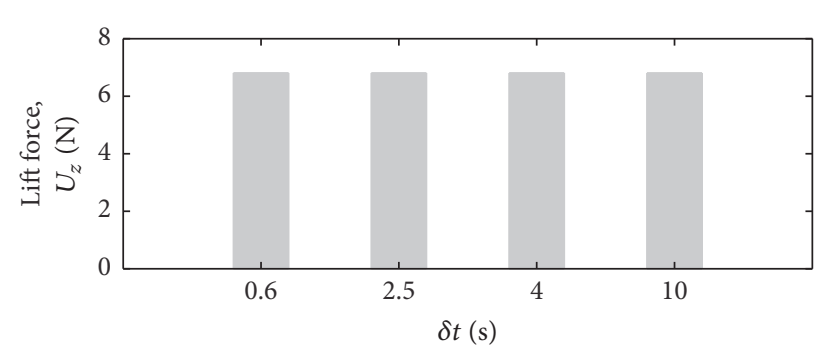

(a)

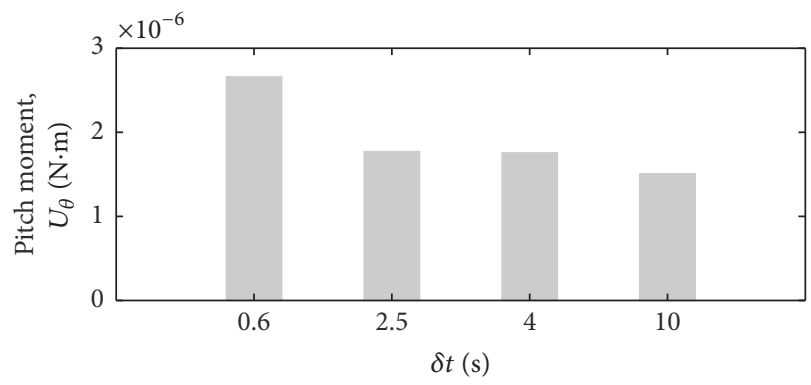

(c)

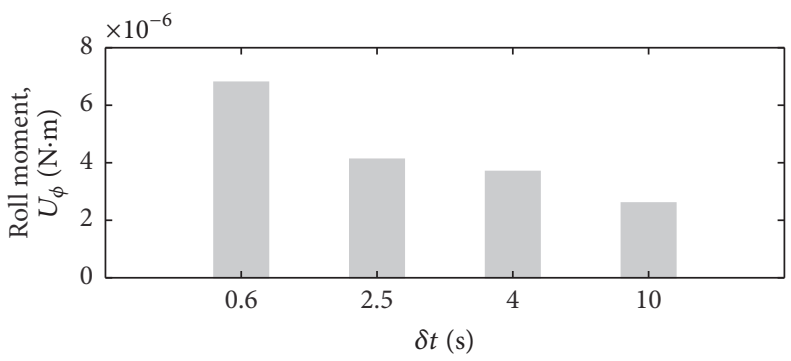

(b)

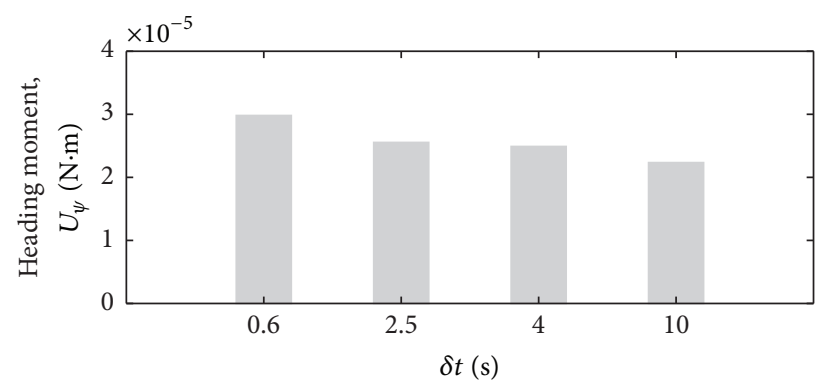

(d)

Figure 15: Mean values of (a) lift force and aerodynamical (b) roll and (c) pitch and (d) heading moments, respectively. 
TABLE 8: Waypoints configuration with their optimal overfly-times schedule.

\begin{tabular}{lccc}
\hline Waypoint & $t_{\text {scenario }_{1}}(\mathrm{~s})$ & $t_{\text {scenario }_{2}}(\mathrm{~s})$ & $t_{\text {scenario }_{3}}(\mathrm{~s})$ \\
\hline Initial & 0 & 0 & 0 \\
1 & 10 & 18.44 & 15 \\
2 & 25 & 34.55 & 28.46 \\
3 & 43 & 47.26 & 45.42 \\
4 & 68 & 60.24 & 63.21 \\
5 & 80 & 71.67 & 75.37 \\
6 & 95 & 89.44 & 94.81 \\
7 & 118 & 105.39 & 113 \\
8 & 130 & 120.76 & 125 \\
Final & 140 & 140 & 140 \\
\hline
\end{tabular}

TABLE 9: Comparison results of induced efforts.

\begin{tabular}{lcccc}
\hline Scenario & Lift force & Roll moment & Pitch moment & \multicolumn{2}{c}{$\begin{array}{c}\text { Heading moment } \\
(\mathrm{N} \cdot \mathrm{m})\end{array}$} \\
\hline 1 & $(\mathrm{~N})$ & $(\mathrm{N} \cdot \mathrm{m})$ & $1.0145 \times 10^{-5}$ & $3.111 \times 10^{-5}$ \\
2 & 6.8166 & $1.5055 \times 10^{-5}$ & $1.3739 \times 10^{-6}$ & $2.4893 \times 10^{-5}$ \\
3 & 6.8092 & $2.5521 \times 10^{-6}$ & $2.631 \times 10^{-6}$ & $2.8259 \times 10^{-5}$ \\
\hline
\end{tabular}

TABLE 10: Waypoints configuration with their optimal overfly-times schedule.

\begin{tabular}{lcccc}
\hline Waypoint & $t_{\delta t=0.6}(\mathrm{~s})$ & $t_{\delta t=2.5}(\mathrm{~s})$ & $t_{\delta t=4}(\mathrm{~s})$ & 0 \\
\hline Initial & 0 & 0 & 0 & 14 \\
1 & 10.6 & 12.5 & 27.39 & 37.87 \\
2 & 25.6 & 27.5 & 47 & 33.67 \\
3 & 43.22 & 45.5 & 64 & 45.48 \\
4 & 67.4 & 65.5 & 76 & 75.48 \\
5 & 79.4 & 77.77 & 95.61 & 92.11 \\
6 & 95.6 & 97.17 & 114 & 110.17 \\
7 & 117.4 & 115.5 & 130.22 & 127.27 \\
8 & 129.4 & 130.45 & 147.54 & 149.46 \\
Final & 144.68 & 146.22 & & \\
\hline
\end{tabular}

TABLE 11: Comparison results of trajectory length and fitness value.

\begin{tabular}{lcr}
\hline$\delta t(\mathrm{~s})$ & Trajectory length $(\mathrm{m})$ & Fitness value \\
\hline 0.6 & 216.29 & $8.1647 \times 10^{3}$ \\
2.5 & 206.21 & $5.5334 \times 10^{3}$ \\
4 & 203.97 & $4.8964 \times 10^{3}$ \\
10 & 203.77 & $4.7394 \times 10^{3}$ \\
\hline
\end{tabular}

TABLE 12: Comparison results of induced efforts.

\begin{tabular}{|c|c|c|c|c|}
\hline$\delta t(\mathrm{~s})$ & $\begin{array}{l}\text { Lift force } \\
(\mathrm{N})\end{array}$ & $\begin{array}{l}\text { Roll moment } \\
\qquad(\mathrm{N} \cdot \mathrm{m})\end{array}$ & $\begin{array}{l}\text { Pitch moment } \\
\qquad(\mathrm{N} \cdot \mathrm{m})\end{array}$ & $\begin{array}{l}\text { Heading moment } \\
(\mathrm{N} \cdot \mathrm{m})\end{array}$ \\
\hline 0.6 & 6.8101 & $6.7975 \times 10^{-6}$ & $2.6577 \times 10^{-6}$ & $2.9807 \times 10^{-5}$ \\
\hline 2.5 & 6.8094 & $4.1188 \times 10^{-6}$ & $1.7692 \times 10^{-6}$ & $2.5535 \times 10^{-5}$ \\
\hline 4 & 6.8093 & $3.6939 \times 10^{-6}$ & $1.7541 \times 10^{-6}$ & $2.4895 \times 10^{-5}$ \\
\hline 10 & 6.8089 & $2.5992 \times 10^{-6}$ & $1.5055 \times 10^{-6}$ & $2.2336 \times 10^{-5}$ \\
\hline
\end{tabular}




\section{Acknowledgments}

This paper is partly supported by the National Natural Science Foundation of China (Grants nos. 61473183 and U1509211), National Postdoctoral Program for Innovative Talents (no. BX201600103), and the China Postdoctoral Science Foundation (no. 2016M601600). The authors would like to thank Bahij Eliker, Zahia Oueldkherroubi, and Nafissa Zouad for their support and helpful feedback for drafting the paper.

\section{References}

[1] M. Huerta, Integration of civil Unmanned Aircraft Systems (UAS) in the National Airspace System (NAS) Roadmap, Federal Aviation Administration, 2013.

[2] L. Lai, C. Yang, and C. Wu, "Time-optimal control of a hovering quad-rotor helicopter," Journal of Intelligent \& Robotic Systems, vol. 45, no. 2, pp. 115-135, 2006.

[3] Y. Bouktir, M. Haddad, and T. Chettibi, “Trajectory planning for a quadrotor helicopter," in Proceedings of the 16th Mediterranean Conference on Control and Automation, pp. 1258-1263, Ajaccio, France, June 2008.

[4] A. Chamseddine, Y. Zhang, and C.-A. Rabbath, "Adaptive trajectory planning for a quad-rotor unmanned aerial vehicle," in Proceedings of the AIAA Guidance, Navigation, and Control Conference, pp. 2-5, Toronto, Canada, August 2010.

[5] A. Chamseddine, T. Li, Y. Zhang, C. A. Rabbath, and D. Theilliol, "Flatness-based trajectory planning for a quadrotor unmanned aerial vehicle test-bed considering actuator and system constraints," in Proceedings of the American Control Conference, pp. 920-925, Montreal, Canada, June 2012.

[6] A. P. Schoellig, F. L. Mueller, and R. D’Andrea, “Optimizationbased iterative learning for precise quadrocopter trajectory tracking," Autonomous Robots, vol. 33, no. 1-2, pp. 103-127, 2012.

[7] E. Kahale, P. Castillo, and Y. Bestaoui, "Minimum time reference trajectory generation for an autonomous quadrotor," in Proceedings of the International Conference on Unmanned Aircraft Systems, pp. 126-133, Orlando, Fla, USA, March 2014.

[8] M. Hehn and R. D’Andrea, "Quadrocopter trajectory generation and control," in Proceedings of the IFAC World Congress, vol. 44, pp. 1485-1491, 2011.

[9] M. Hehn, R. Ritz, and R. D’Andrea, "Performance benchmarking of quadrotor systems using time-optimal control," Autonomous Robots, vol. 33, no. 1-2, pp. 69-88, 2012.

[10] M. Hehn and R. Dandrea, "Real-Time Trajectory Generation for Quadrocopters," IEEE Transactions on Robotics, vol. 31, no. 4, pp. 877-892, 2015.

[11] J. Jamieson and J. Biggs, "Path planning using concatenated analytically-defined trajectories for quadrotor UAVs," Aerospace, vol. 2, no. 2, pp. 155-170, 2015.

[12] D. Mellinger and V. Kumar, "Minimum snap trajectory generation and control for quadrotors," in Proceedings of the IEEE International Conference on Robotics and Automation, pp. 2520 2525, IEEE, Shanghai, China, March 2011.

[13] D. Mellinger, N. Michael, and V. Kumar, "Trajectory generation and control for precise aggressive maneuvers with quadrotors," International Journal of Robotics Research, vol. 31, no. 5, pp. 664674, 2012.

[14] J. Yu, Z. Cai, and Y. Wang, "Optimal trajectory generation of a quadrotor based on the differential flatness," in Proceedings of the 28th Chinese Control and Decision Conference, pp. 678-683, Yinchuan, China, March 2016.

[15] K. Eliker, H. Bouadi, and M. Haddad, "Flight planning and guidance features for an UAV Flight Management Computer," in Proceedings of the 21st IEEE International Conference on Emerging Technologies and Factory Automation, 6, 1 pages, Berlin, Germany, September 2016.

[16] I. D. Cowling, O. A. Yakimenko, J. F. Whidborne, and A. K. Cooke, "Direct method based control system for an autonomous quadrotor," Journal of Intelligent \& Robotic Systems, vol. 60, no. 2, pp. 285-316, 2010.

[17] A. Chamseddine, Y. Zhang, C. A. Rabbath, C. Join, and D. Theilliol, "Flatness-based trajectory planning/replanning for a quadrotor unmanned aerial vehicle," IEEE Transactions on Aerospace and Electronic Systems, vol. 48, no. 4, pp. 2832-2848, 2012.

[18] S. Taamallah, X. Bombois, and P. M. J. Van den Hof, “Trajectory planning and trajectory tracking for a small-scale helicopter in autorotation," Control Engineering Practice, vol. 58, pp. 88-106, 2017.

[19] F. Morbidi, R. Cano, and D. Lara, "Minimum-energy path generation for a quadrotor UAV," in Proceedings of the 2016 IEEE International Conference on Robotics and Automation, pp. 14921498, Stockholm, Sweden, March 2016.

[20] M. De Lellis Costa de Oliveira, Modeling, identification and control of a quadrotor aircraft [M.S. thesis], Faculty of Electrical Engineering, Prague, Czech Republic, 2011.

[21] L. Derafa, T. Madani, and A. Benallegue, "Dynamic modelling and experimental identification of four rotors helicopter parameters," in Proceedings of the IEEE International Conference on Industrial Technology (ICIT '06), pp. 1834-1839, Mumbai, India, December 2006.

[22] H. Bouadi and F. Mora-Camino, "Modeling and adaptive flight control for quadrotor trajectory tracking," Journal of Aircraft, vol. 55, no. 2, pp. 666-681, 2018.

[23] G. G. Rigatos, Nonlinear Control and Filtering Using Differential Flatness Approaches: Applications to Electromechanical Systems, Springer, Cham, Switzerland, 2015.

[24] L. Biagiotti and C. Melchiorri, Trajectory Planning for Automatic Machines and Robots, Springer, Berlin, Germany, 2009.

[25] T. Coleman, M. A. Branch, and A. Grace, Optimization Toolbox: for Use with Matlab, Matlab The Mathworks Inc., 1999. 


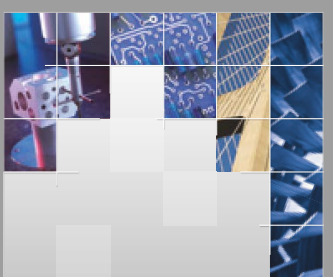

\section{Enfincering}
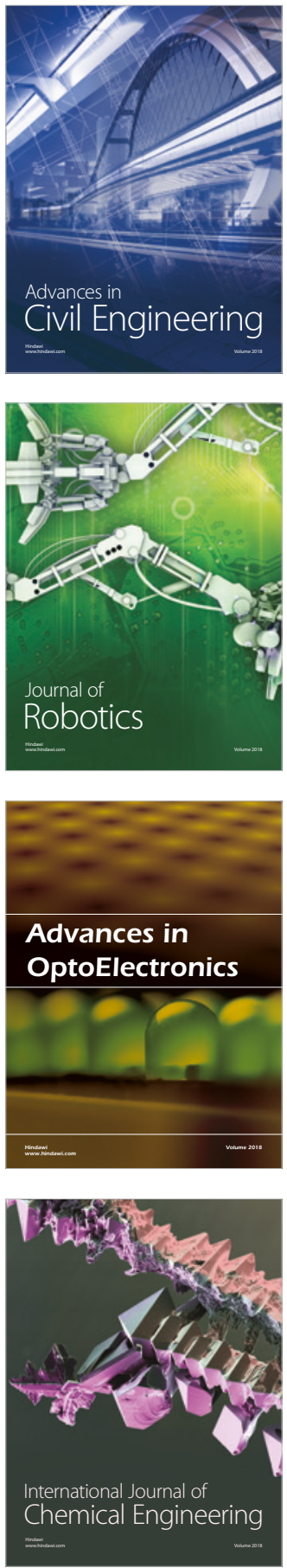

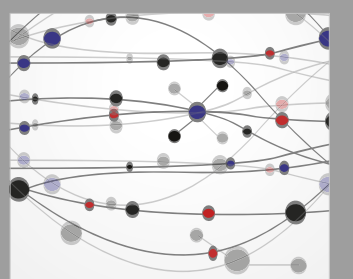

\section{Rotating \\ Machinery}

The Scientific World Journal

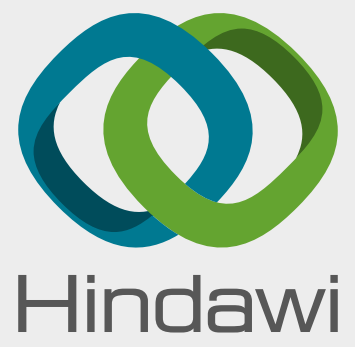

Submit your manuscripts at

www.hindawi.com
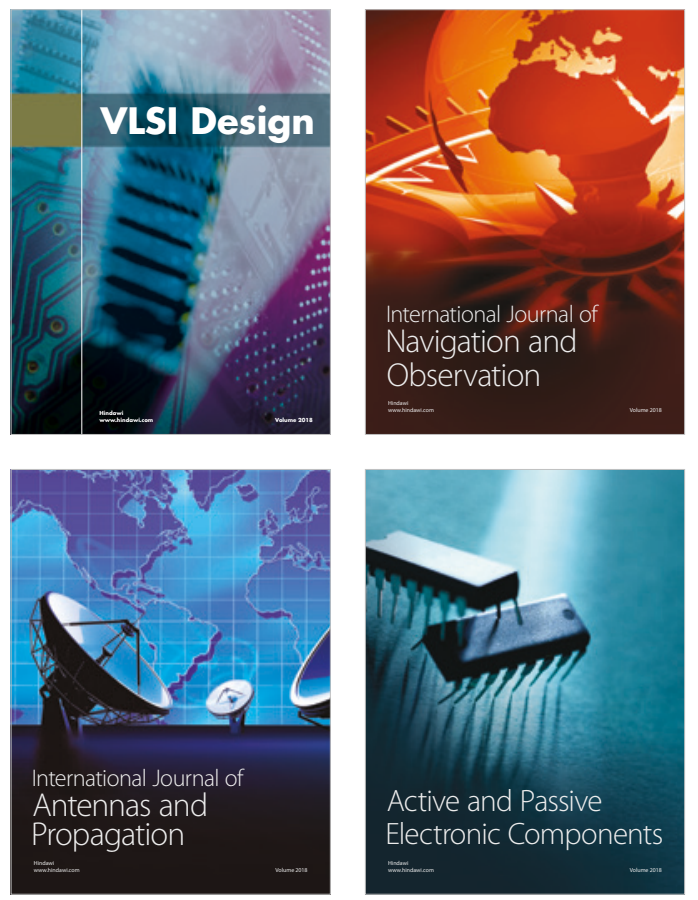
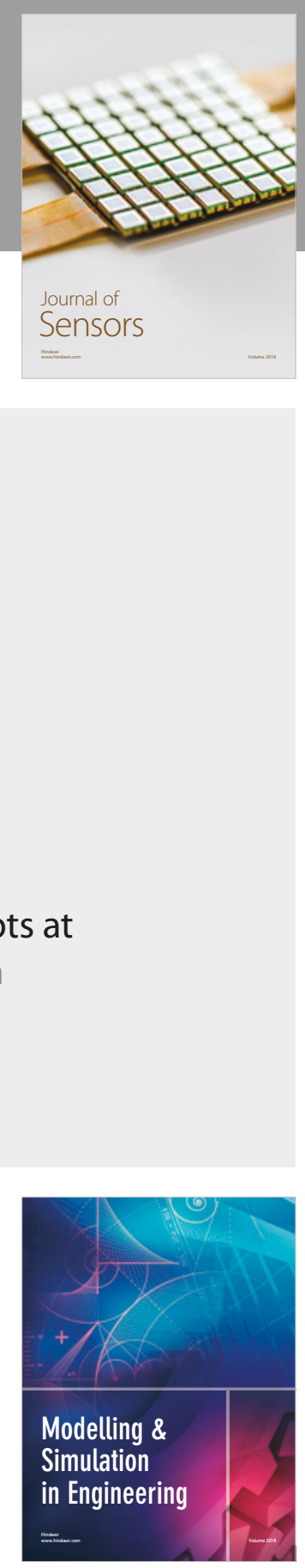

\section{Advances \\ Multimedia}
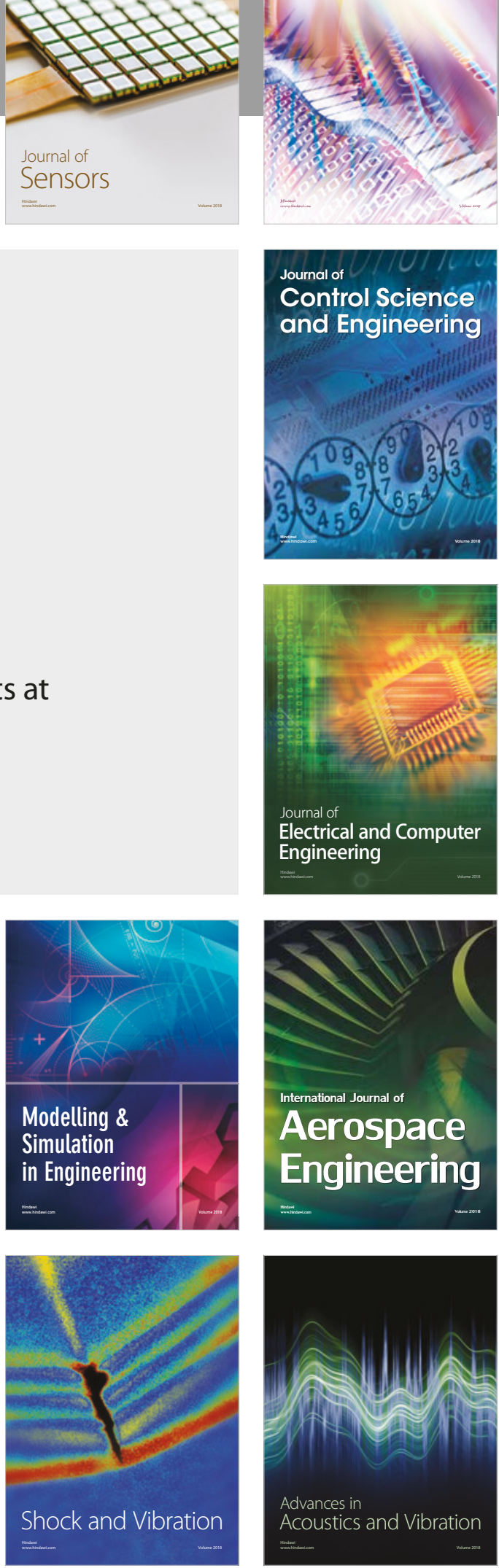